\title{
Plastic Deformation in Nanoindentation of Tantalum: A New Mechanism for Prismatic Loop Formation
}

\author{
T.P. Remington ${ }^{\text {a }}$, C.J. Ruestes ${ }^{\text {b }}$, E. M. Bringa ${ }^{\text {b,c }}$, B. A. Remington ${ }^{\text {d }}$, C.H. Lu ${ }^{\text {a }}$, B. Kad ${ }^{\text {a }}$ and \\ M.A. Meyers ${ }^{\text {a }}$ \\ ${ }^{a}$ University of California, San Diego, La Jolla, CA 92093, USA \\ ${ }^{\mathrm{b}}$ Instituto de Ciencias Básicas, Universidad Nacional de Cuyo, Mendoza 5500, Argentina \\ ${ }^{\mathrm{c}}$ CONICET, Mendoza 5500, Argentina \\ ${ }^{\mathrm{d}}$ Lawrence Livermore National Laboratory, Livermore, CA 94550, USA
}

\begin{abstract}
The mechanisms of deformation under a nanoindentation in tantalum, chosen as a model BCC metal, are identified and quantified. Molecular dynamics simulations and indentation experiments are conducted for [100], [110], and [111] surface orientations. The simulated plastic deformation proceeds by the formation of nanotwins, which rapidly evolve into shear dislocation loops. It is shown through a dislocation analysis that an elementary twin (3 layers) is energetically favorable for a diameter below $\sim 7 \mathrm{~nm}$, at which point a shear loop comprised of a perfect dislocation is formed. MD simulations show that shear loops expand into the material by the advance of their edge components. Simultaneously with this advance, screw components of the loop cross slip and generate a cylindrical surface. When opposite segments approach, they eventually cancel by virtue of the attraction between them, forming a quasi-circular prismatic loop composed of edge dislocation segments. This 'lasso' like mechanism by which a shear loop transitions to a prismatic loop is identified for both [001] and [111] indentations. The prismatic loops advance into the material along <111> directions, transporting material away from the nucleation site. Analytical calculations supplement molecular dynamics and experimental observations, and provide a framework for the improved understanding of the evolution of plastic deformation under a nanoindenter. Dislocation densities under the indenter are estimated experimentally $\left(\sim 1.2 \times 10^{15} \mathrm{~m}^{-2}\right)$, by molecular dynamics $\left(\sim 7 \times 10^{15} \mathrm{~m}^{-2}\right)$, and through an analytical calculation $\left(2.6-19 \times 10^{15} \mathrm{~m}^{-2}\right)$. Considering the assumptions and simplifications, this agreement is considered satisfactory. MD simulations also show expected changes in pile-up symmetry after unloading, compatible with crystal plasticity.
\end{abstract}




\section{Introduction}

The origins of hardness testing can be traced back to the $19^{\text {th }}$ century [1-3]. This simple method to evaluate the strength of metals has been immensely successful, principally because of its simplicity and quasi non-destructive nature. The number obtained, the 'hardness', represents the resistance of the material to penetration, a reasonable measure of compressive strength under lateral confinement. Portable units to measure the hardness are available, and it is also very useful as a research tool, providing a ranking of materials. The plastic deformation under the indenter is highly heterogeneous, and attempts to correlate the numbers obtained with fundamental materials parameters often fall short [4]. Nevertheless, the simple Tabor [5] relationship between yield stress and hardness $\left(\sigma_{y}=H / 3\right)$ is often used, in spite of its limitations.

Nanoindentation testing has gained global acceptance as a tool to probe the mechanical properties of materials at the micrometer and sub-micrometer scale and the continuous loadpenetration curve provides the elastic modulus and hardness through the widely used OliverPharr analysis [6].

In parallel with plasticity analysis of the plastic deformation under an indenter [7-10], molecular dynamics (MD) simulations are becoming quite realistic and capture, albeit at a much smaller spatial scale and much larger indentation velocities, the features of the plastic deformation processes occurring under the indenter. There is a large number of studies carrying simulations for face centered cubic (FCC) metals, due to the large number of experiments for such metals, but also due to the availability of empirical potentials for FCC metals which behave reasonably well at large strains. Zhu et al. [11], Li et al. [12], and van Vliet et al. [13] performed MD and finite element calculations for FCC metals and showed that stacking-fault loops nucleated under the indenter, not at the surface, but below it. Although there are several dislocation nucleation criteria [11], a simple criterion based on a threshold shear stress is often used. Indeed, the region of maximum shear stress under an indenter is below the surface. The Hertz lines of maximum shear stress in the compression of a flat surface by a cylindrical or spherical indenter show this clearly [14]. Zhong and Zhu [15] showed that nucleation, gliding, and interaction of Shockley partial dislocations in FCC structures were involved in the early plastic stages of indentation, and recently detailed dislocation analysis was also carried out by Begau et al. [16] and Engles et al. [17]. 
Scanning tunneling microscopy and Atomic Force Microscopy (AFM) in ultrahigh vacuum has also been used to characterize indentation in gold (FCC structure) [18]. There are many related studies on Au nanoindentation [19]. Elastic and plastic indentations were identified both in the residual impression images and by features in their force-displacement curves such as the sink-in depth, pop-ins, and hysteresis energy but there are still many open questions [20].

Fang et al. [21] performed MD calculations for aluminum and reported partial dislocations emanating from the indentation site, with stacking faults on $\{111\}$ planes. Tsuru et al. [22] also investigated the behavior of aluminum, using both atomistic and microscopic models. In general, it is often found that the necessary critical resolved shear stresses for dislocation nucleation is higher than the theoretical shear strength because of the compressive stress state underneath the indenter, with load-displacement curves showing both elastic and pop-in displacements. Begau et al. [23] performed simulations for copper, allowing the dislocations to move larger distances from the indentation region and observed leading and trailing partial dislocations and stacking faults between them in configurations fairly similar to the ones observed by Traiviratana et al. [24] in the growth of voids in copper. Ziegenhain et al. [25] investigated the effect of crystal anisotropy (in $\mathrm{Cu}$ and $\mathrm{Al}$ ) on the generation of partial and perfect dislocations and observed emission of prismatic loops below the indenter.

In one of the first MD simulations for indentation of body-centered cubic (BCC) metals, tungsten was investigated using a Finnis-Sinclair potential with approximately 850,000 atoms, [111] and [110] surfaces, and a tip diameter of $10 \mathrm{~nm}$ [26]; penetration depths were limited due to the relatively small sample size, but allowed the observation of the earlier stages of plastic activity, often including emission of prismatic loops. Kumar et al. [27], recently simulated Fe, pure and with impurities, using about 1 million atoms, a $4 \mathrm{~nm}$ diameter indenter, and velocity of $100 \mathrm{~nm} / \mathrm{ps}$, showing that slip occurs in $\{110\},\{112\}$ and $\{123\}$ planes, as expected from BCC crystal symmetry.

Alcalá et al. [28] described dislocation structures under nanoindentations in tantalum crystals using the potential by Li et al. [29], and presented experimental loading curves for [001], [011] and [111] surfaces. They found that (indent depth)/(indenter diameter) 0.2 gave a reasonable value for the first pop-in event in both simulations and experiments. They reported the generation of stacking faults and twins directly under the indenter as well as dislocation loops; twin nucleation and interaction as well as annihilation produced these loops. Their load- 
penetration curves showed a marked elastic-plastic transition in line with their experimental measurements for [100], [110], and [111], which exhibited an elasto-plastic pop-in corresponding to the emission of the first dislocations. The leading edges of the loops were of edge character and the trailing parts were screw dislocations. Twin annihilation was attributed to reduction of stacking fault energy for twin layers thinner than four atomic layers and found to be enhanced at higher temperatures and decreased loading rates. Sectional views showed the progressive development of dislocation loops and their interactions.

It should be mentioned that most indentation studies are either computational or experimental. The investigations conducted by Alcalá [28], Lodes et al. [30] or Sadrabadi et al. [31] on $\mathrm{CaF}_{2}$ are rare exceptions. Since this crystal is amenable to etch pitting, Lodes et al. [30] were able to quantitatively estimate dislocation densities under the indenter and compare them with MD predictions. The molecular dynamics predictions essentially confirmed earlier experimental results [31]: dislocation density decreased with penetration depth consistently with a decrease in hardness due to increasing load.

The objective of this contribution is to provide a combined computational-experimentalanalytical study leading to a quantitative knowledge of the mechanisms of plastic deformation under the indenter for a model BCC metal, tantalum. Below we first describe experimental and computational methods, then present loading curves and AFM images of the indented surfaces. We present simulated loading and unloading curves, describing in detail the formation of prismatic loops during loading by a new "lasso" mechanism and present a dislocation-based model for that loop formation. Finally, we present simulated pile-ups for different surface orientations and show dislocation densities from experiments, an analytical model and MD simulations.

\section{Experimental and computational methods}

\subsection{Experimental materials and procedures}

All tantalum single crystals were received after being mechanically and electromechanically polished by Surface Preparation Laboratory (a company in the Netherlands) to attain a surface roughness less than $\sim 1 \mathrm{~nm}$. Load controlled nanoindentation experiments were performed using a Ubi 1 nano-mechanical test instrument (Hysitron, Inc., Minneapolis, MN) equipped with a diamond Berkovich tip having a $\sim 100 \mathrm{~nm}$ nominal radius of curvature. For each 
Ta sample, two grids of $10 \times 10$ indentations were made, one using a loading rate of $30 \mu \mathrm{N} / \mathrm{sec}$ with a maximum load of $150 \mu \mathrm{N}$, and the other with a loading rate of $200 \mu \mathrm{N} / \mathrm{sec}$ and a maximum load of $1000 \mu \mathrm{N}$. Values of hardness and reduced modulus were calculated from loaddisplacement curves using the Oliver-Pharr method. Prior to performing each indentation, drift was digitally monitored for 40 seconds and found to be less than $0.1 \mathrm{~nm} / \mathrm{sec}$. All tests were performed at $20^{\circ} \mathrm{C}$ in an environmental chamber on an active vibration isolation table.

\subsection{Computational methods}

MD simulations were done using LAMMPS [19]. Indentation was carried out using a rigid spherical indenter tip [32]. Although the Berkovich tip is typically used in nanoindentation studies, it should be noted that such an indenter has a rounded tip of up to $150 \mathrm{~nm}$; therefore, it can be expected that the spherical tip used in our studies renders the same plasticity mechanisms produced by a Berkovich tip prior to significant effects produced by the pyramidal planes. Simulated box size, and tip diameter and velocity were varied within the limits allowed by our computational resources, to ensure our results were not affected by finite-size effects or indentation rate. A detailed parametric study will be published separately, since here we are focusing on large-scale load and unload simulations to understand prismatic loop formation and pile-ups.

We used an indenter diameter of $25 \mathrm{~nm}$ for the [001] surfaces, and $20 \mathrm{~nm}$ for [011] and [111] surfaces. Changing indenter diameter in the range 10-25 $\mathrm{nm}$ did not change the qualitative features of our description below. The MD simulation domain had dimensions of $90 \times 90 \times 66$ $\mathrm{nm}^{3}$ (around 30 million atoms) with an indenter of $25 \mathrm{~nm}$ diameter for indentation of the [001] surfaces; $50 \times 50 \times 40 \mathrm{~nm}^{3}$ (around 6 million atoms) for indentation of the [011] surfaces; and 50 x $50 \times 40 \mathrm{~nm}^{3}$ (around 6 million atoms) for indentation of the [111] surfaces. These sizes are larger than usual in most current indentation simulations, and ensure that the plastic region is properly contained within the simulated volume. It is customary to expect a plasticity zone of up to 3.5 times the radius of the indentation imprint, as a worst-case scenario [33]. As a further check at the end of our simulations, we verified that our box size for the [001] indentation is 6 times the radius of the imprint in the $\mathrm{z}$ direction and 8 times the radius of the imprint in the $\mathrm{x}$ and y directions. 
Indentation was carried along the $\mathrm{z}$ direction, and periodic boundary conditions were applied in $\mathrm{x}-\mathrm{y}$ directions. The sample was energetically minimized and equilibrated at $300 \mathrm{~K}$ and a Langevin thermostat was applied to the sides and bottom of the domain in order to dampen possible boundary effects. Unlike nanoindentation experiments, in which the test is performed in what is called load-control mode, MD simulations are typically done in a displacement control by applying a constant penetration rate to the indenter [32].

An indentation velocity of $34 \mathrm{~m} / \mathrm{s}$ was used, compared to $100 \mathrm{~m} / \mathrm{s}$ in Kumar et al. [27], 20 $\mathrm{m} / \mathrm{s}$ in Lodes et al. [30] and 40-0.004 m/s in Alcalá et al. [28]. This velocity is approximately $1 \%$ of the bulk sound velocity $[32,34]$, and considered sufficiently low to minimize dynamic effects. Reducing the indentation velocity to $3.4 \mathrm{~m} / \mathrm{s}$ did not produce any qualitative change in loading curves and plasticity, but lowered slightly the plastic threshold, as expected. Quasistatic simulations as in Hagelaar et al. [26] might lead to somewhat different results. Of course, simulated speeds are orders of magnitude larger than experimental values, and there might be effects due to the high strain rate of every MD simulation, for instance in dislocation-twinning competition [35].

It is challenging to develop empirical potentials for BCC metals which would work at the

large strains and stresses reached in indentation simulations [36]. Here we used a recently presented EAM potential by Ravelo et al. [37], which has been specifically designed to work at high stress and describes well the elastic properties of Ta up to high pressures, alongside properties associated with plasticity, like shear stress-strain curves and gamma energy curves [37]. This potential has been recently shown to describe extremely well the microstructure induced by high pressure loading of Ta [33].

Defective structures were filtered by means of Common Neighbor Analysis [38] and visualized by means of OVITO [39]. Dislocation line lengths were computed using the Dislocation eXtraction Algorithm (DXA) [40], and dislocation density can then be defined for a given volume containing those dislocations.

\subsection{Experimental sample characterization}

The nanoindented surfaces were observed by scanning electron microscopy (SEM) to identify the location of the nanoindented matrices. A FEI XL30 Ultra High Resolution (UHR) SEM was used at the Nano3 facility in the University of California, San Diego, with a resolution 
of $\sim 1 \mathrm{~nm}$ possible at $10 \mathrm{KV}$ or higher and $1.7 \mathrm{~nm}$ at $1 \mathrm{KV}$. Subsequent to atomic force microscopy (AFM) was used to image the surface profiles of the pile-ups and indentation depths. A Veeco scanning probe microscope with a $125 \mu \mathrm{m}$ x $125 \mu \mathrm{m}$ non-magnetic scanner and $5 \mu \mathrm{m}$ vertical range was used, operating in the tapping mode and scanning the samples topographically. The AFM tips used were aluminum coated NCHR silicon with thickness of $4 \mu \mathrm{m}$, length $125 \mu \mathrm{m}$, width $30 \mu \mathrm{m}$, resonance frequency of $320 \mathrm{kHz}$ and force constant of $42 \mathrm{~N} / \mathrm{m}$. The specimens for transmission electron microscopy (TEM) were prepared by focused ion beam (FIB) milling. FIBing was conducted at two facilities: University of California, Los Angeles and Oak Ridge National Laboratory. The Nanoelectronics Research Facility (NRF) at UCLA uses a Nova 600 SEM/FIB system to produce TEM foils. The FIB has a $10 \mathrm{~nm}$ resolution and 5-axis Stage with $150 \mathrm{~mm}$ travel. The Shared Research Equipment User Facility (ShaRE) at ORNL uses a FEI Nova 200 dual-beam SEM/FIB system, with $7 \mathrm{~nm}$ resolution capability. Samples were coated with platinum before milling. TEM foils were cut using FIB milling perpendicular to the surface of the specimen, each foil containing 2-3 cross sections of nanoindentations. Foils were cut to a thickness of around $\sim 50 \mathrm{~nm}$. Observation was carried out at the ORNL ShaRE facility using a Philips CM200-FEG TEM-STEM in the two-beam condition.

\section{Results and discussion}

\subsection{Experimental measurements and characterization}

During the nanoindentation process, the applied load and corresponding indentation depths were recorded for each individual indent. From this data an average load versus indentation depth profile was obtained for all three orientations [100], [110] and [111] of Ta. Figure 1 shows the elastic Hertzian curve and the loads at which the different orientations transition into plastic deformation. The first emission of dislocations ("pop-in") marks the beginning of significant deviation from the elastic Hertzian curve. Tantalum orientations [100] and [110] transition from the elastic to plastic regime at a lower load of $\sim 150 \mu \mathrm{N}$ than orientation [111], which transitions from elastic to plastic deformation at a load of $\sim 200 \mu \mathrm{N}$. These load-

displacement (F-h) curves are similar to the ones in the work done by Biener et al. [41], and by Alcalá et al. [28] for the same orientations.

After completing the nanoindentation, AFM images were taken, showing plastic deformation pile-ups around the edges of the indentations. The AFM image for Ta [100] done by 
Biener et al. [41] using a Berkovich tip correlates well to our AFM results, Fig. 2a. Figures 2c,e show the pile-ups for [110] an [111]. Due to the Berkovich tip geometry the pile-ups form around the perimeter of the nanoindentation. Biener et al. [41] used a spherical indenter tip for nanoindented Ta [100], and observed four pile-ups forming along the diagonal <110> orientations. The pile-ups observed with the Berkovich indenter are primarily dictated by the pyramid faces and not by the anisotropy of plastic deformation of the crystal. The vertices of the triangular indentation are fairly flat, while the sides rise to account for volume conservation. Figures $2 \mathrm{a} 2 \mathrm{~b}$ and $3 \mathrm{a}$ show a white streak that is an artifact of the AFM. However, the profiles are correct.

Depth profiles of the indentations were obtained and typical depth profiles of a nanoindentation showing pile-ups at the surface perimeter of the indents is shown in Figs. 2b, $2 \mathrm{~d}$ and $2 \mathrm{f}$. The pile-up heights are largest in the middle of the face and smallest at the vertices. The average pile-up height around the nanoindentations for all three single crystal orientations [100], [110] and [111] is within the range of $h_{p}=5-8 \mathrm{~nm}$ for a load of around $1,000 \mu \mathrm{N}$.

FIB was used to cut and polish samples for TEM characterization from the center portion of an indentation and immediately analyzed under TEM double tilt condition. TEM images were taken at indentation sites as well as far away from nanoindentations. Dislocations densities under and around the nanoindentations were calculated from TEM images using the linear intercept method. Figures 3 and 4 show a cross-sectional area of a nanoindentation for [100] and [111] Ta, respectively. The average dislocation density under the nanoindentation for the[100] crystal was calculated to be $(1.2 \pm 0.5) \times 10^{15} \mathrm{~m}^{-2}$. This dislocation density was calculated for a two-beam condition in which the largest possible number of dislocations was imaged. Density was calculated by using a rectangular grid obtaining the number of intercepts in an area of $235,200 \mathrm{~nm}^{2}$. The volume was calculated assuming a thickness of $50 \mathrm{~nm}$ for the TEM foil, the specified thickness during the FIB process. FIBing was attempted directly at the center of each nanoindentation; however, it is a very difficult process, resulting in TEM foils with segments of indentations a little off from its center. This is the case for Figure 4, and it can be seen that the indentation is significantly smaller than the one shown in Figure 3. Therefore, the calculated dislocation density for this specimen would be somewhat speculative. Defects due to FIB milling do include point defects (interstitials, vacancies), dislocations and inclusion of amorphous material into the TEM foil, but the exact value for these defects is not known for tantalum and 
many other materials. In light of the possibility of the FIB process adding defects into the TEM foil, a reference FIB sample was cut far away from nanoindentation sites and compared to a FIB cut taken right at an indentation. Using the reference sample as a basis for identifying FIB induced defects, the dislocation density calculated right under the nanoindentation did not include dislocations introduced possibly by the milling process.

\subsection{Atomistic modeling of plasticity}

The evolution of dislocations under the indenter is shown in the sequence of Figure 5 for a (001) surface. It proceeds by initial formation of planar defects in a manner akin to that described by Alcalá et al. [28]. The use of the Ravelo et al. potential [34, 37] in the current calculations, instead of the Li et al. potential [29] applied by Alcalá et al. [28], results in smaller twinned regions. The twins are $\{112\}$ twins, as expected for BCC metals under compressive stress. However, the overall evolution is similar. We note that twinning often appears under high strain rate conditions, and the twinning observed in our simulations might not appear for indent velocities approaching experimental values, which are outside the reach of current large scale atomistic simulations.

Shear loops form and propagate along the expected <111> slip directions. Two of the four

directions are marked in Fig. 5. The shear loops expand by the advance of the edge components, while the screw components can, and do, undergo limited cross slip. This cross slip of the screw components eventually leads to a pinching off action, which produces prismatic loops. It is noted that the edge components cannot cross slip.

In order to focus on the formation of the prismatic loops in detail, a section of the sample was isolated and it is shown in Fig. 6. The screw components are straight whereas the edge component forms the front of the loop. As the edge component advances, the screw components cross slip and eventually reencounter each other (Fig. 6e), canceling in the process (Fig. 6f). Thus, a prismatic loop is formed.

A similar evolution was observed for indentation along [111]. The shear loops in Fig. 7a propagate with a Burgers vector of $b / 2[111]$. The screw components of the loops are perpendicular to the surface. One of these loops undergoes the 'pinching-off' of a prismatic loop through the cross-slip action of the screw components as the edge component advances. In Fig. 7d, a loop is piched off. The formation of two prismatic loops is shown in Fig. 7f. As the indenter penetrates into the sample additional prismatic loops are formed along this and other 
orientations. It should be noted that Hagelaar et al. [26] also observed prismatic loop punching during loading for the [111] indentation, and associated the loop formation with shear in their atomistic indenter.

The process of deformation under the indenter is schematically rendered in Fig. 8 for a generic [111] direction. The initial formation of one single loop having a [111] slip direction (Fig. 8a) is followed by cross slip of the screw components as the front advances (Figs. 8b-e). Two families of slip planes having a common [111] direction contribute to this: $\{110\}$ and $\{211\}$. The screw components, in a 'lasso' action, close in to form a circular edge front. The attraction between the opposite screw segments is due to the fact that the dislocation lines have opposite signs, while their Burgers vectors are parallel. In Figure 8f, the two screw components of the loop attract each other and cancel. This leads to the pinching off effect, which is complete in Fig. $8 \mathrm{~g}$, marking the full emission of a prismatic loop taking an almost circular shape. This prismatic loop subsequently advances, and the process repeats itself as deformation proceeds.

In this study, all the observed prismatic loops had shapes that were close to circular. The shear loops that gave rise to the prismatic loops, on the other hand, were approximately rectangular, with two lateral components composed of screw dislocations, and the advancing front, which had some curvature, composed of dislocation segments of primary edge character. However, if fewer slip planes are involved, the prismatic loops can have triangular or hexagonal shapes. Indeed, this was observed in MD simulations by Tang et al. [35] for the growth and collapse of nanoscale voids in Ta. Figure 9c from Tang et al. [35] does show the final stages of the pinching-off leading to the lasso loop formation. It is interesting to notice that Figure 6 of Lodes et al. [30], show something indicative of a lasso mechanism for a cubic ionic crystal, with an incomplete lasso (loop marked 1), that has evolved into a prismatic loop in 2.

As the plastic deformation region below the indentation develops, a pile-up is formed due to volume conservation. The volume displaced by the indenter is partially extruded upwards. This pile-up region is also experimentally observed (see Section 3.1). The pile-up follows the corresponding symmetry of the crystal slip. For instance, for [001] indentation, there are four $<111>$ slip directions leading to four-fold symmetry of the surface pile-ups. Figure 10a shows a top view of the sample, with the four directions of loop emission indicated, and the pile-ups shown in Fig. 10b. The side view of the pile-ups is shown in Fig. 10c. The height of the pile-ups, $\mathrm{h}_{\mathrm{p}}$, is between 2.5 and $4.5 \mathrm{~nm}$, as shown in Fig 9c. 
Similar evolution of dislocations leading to prismatic loop formation was observed for the [011] and [111] orientations, as shown in Fig. 11. The geometry of pile-ups is the same as predicted by crystal plasticity finite element model (FEM) simulations [42]. Those simulations give perfectly symmetric pile-ups, while MD gives some asymmetry due to small thermal and stress fluctuations. In addition to prismatic loops, "half loops" attached to the surface are moving away in Fig. 11b. These are loops that moved towards the surface, which absorbed part of the loops without pinning them. Something similar was observed by Lodes et al. [30], for different planes, but with a similar mechanism.

\subsection{Plasticity nucleation model}

There are many studies on plasticity initiation using simulation and models [12]. We recreate here a simple model to apply to our simulations. Plastic deformation, as mentioned earlier, does not start at the surface, but below it. At first, this seems counter-intuitive. However, the MD calculations show that the maximum shear stress is not at the surface but below it [12]. Figure 12a shows that the emission of planar defects starts below the surface. The maximum shear stress regions are marked in Fig. 12b and show that the maximum value (about $10 \mathrm{GPa}$ ) is at $\mathrm{z} \approx 0.5 \mathrm{a}$, where $\mathrm{a}$ is the radius of the imprint. This can be corroborated by Hertz's calculations [43], as will be demonstrated below. The stresses below a rigid sphere pressing against a plate have been calculated by Hertz. The principal stresses under the indenter are:

$$
\begin{aligned}
& \sigma_{1}=-F\left\{\left[1-\left|\zeta_{a}\right| \tan ^{-1}\left(\frac{1}{\left|\zeta_{a}\right|}\right)\right](1-v)-\frac{1}{2\left(1+\xi_{a}^{2}\right)}\right\} \\
& \sigma_{2}=-\frac{F}{1+\zeta_{a}^{2}}
\end{aligned}
$$

where,

$$
\zeta_{a}=\frac{z}{a}
$$

This is a normalized distance. The contact area of the sphere on the plate is. The maximum shear stress is: 


$$
\tau_{\max }=\frac{\sigma_{1}-\sigma_{2}}{2}
$$

According to the Hertz analysis for a rigid sphere of radius R (acting against a plate with Young's modulus E and Poisson's ratio v), is given by:

$$
a=\left[\frac{3}{8} \frac{\left(1-v^{2}\right)}{E} 2 R F\right]^{1 / 3}
$$

where $\mathrm{R}$ is the sphere radius and $\mathrm{F}$ the force applied on the plate [44]. Figure 12c shows the variation of the maximum shear stress divided by the pressure $\mathrm{P}$ (equal to the force $\mathrm{F}$ divided by the contact area) for tantalum, as a function of depth $\mathrm{z}$, normalized to a $(\mathrm{z} / \mathrm{a}=\mathrm{z})$. It can be seen that it reaches a maximum at a depth $\mathrm{z} \sim 0.66$, from the Hertzian equations. The results of MD are similar, giving $\mathrm{z} \sim 0.5$. The value of the critical stress is $(0.28 \mathrm{G}) \sim 19 \mathrm{GPa}$ using $\mathrm{G}=69 \mathrm{GPa}$ for the Ravelo potential $[34,37]$. This is nearly twice the MD value. Given the simplicity of the model, this difference is still reasonable.

\subsection{Analytical model for the transition from twins to perfect dislocations}

Plastic deformation initiates by the formation of twins. Upon expansion, they generate perfect dislocations. This was also observed by Tang et al. [35] and Alcalá et al. [28], and is also shown in the sequence of Fig. 13. We provide below an energetic argument for this transition. It is based on the classic equation for the critical size of a shear loop as a function of the applied shear stress. The treatment is given by Hull and Bacon [44] and was extended to perfect dislocations and stacking faults by Meyers et al. [45].

We consider two alternative scenarios: the nucleation of a shear loop of dislocations with $b / 2<111>$ and the nucleation of a three-layered twin with three twinning dislocations $b / 6<111>$. They are shown in Figs. 14a and 14b, respectively, and produce the same amount of displacement. The energy of a perfect dislocation loop of radius $r$ can be obtained in a simplified manner assuming that one half $(\pi r)$ has screw character and the other half edge character. Making a cutoff at 2 r, we have:

$$
E_{e l}=\frac{r G b^{2}}{4}\left(1+\frac{1}{1-v}\right) \ln \frac{2 r}{r_{0}}
$$

where $r_{0}$ is the core radius. The work done by the formation of the loop is:

$W=\tau b \pi r^{2}$ 
The total change in energy is:

$\Delta E=E_{e l}-W=\frac{r G b^{2}}{4}\left(1+\frac{1}{1-v}\right) \ln \frac{2 r}{r_{0}}-\tau b \pi r^{2}$

Taking the derivative of the total energy and making it equal to zero gives the critical radius:

$$
\begin{aligned}
& \frac{\partial \Delta E}{\partial r}=0 \\
& r_{c}=\frac{G b}{8 \pi \tau}\left(\frac{2-v}{1-v}\right)\left[\ln \left(\frac{2 r_{c}}{r_{0}}\right)+1\right]
\end{aligned}
$$

Repeating the procedure for a twin, we have to add a term to account for the two twin boundaries formed, with energy per unit area equal to $\gamma_{T}$ :

$$
\Delta E=\frac{3 r G b_{T}}{4}\left(\frac{2-v}{1-v}\right) \ln \frac{2 r_{T}}{r_{0}}+2 \pi r^{2} \gamma_{T}-3 \pi b_{T} \pi r_{T}^{2}
$$

The Burgers vector of the partial dislocation is $b_{T}$ and the twin-boundary energy is $\gamma_{T}$. Through the application of Eq. 8 this leads to:

$$
r_{c T}=\frac{3 G b_{T}^{2}}{8 \pi\left(3 \tau b_{T}-2 \gamma_{T}\right)}\left(\frac{2-v}{1-v}\right)\left[\ln \left(\frac{2 r_{c T}}{r_{0}}\right)+1\right]
$$

The critical radius is $r_{c T}$. Figure 14c shows a plot of the critical radii for twin and shear loop formation as a function of the applied shear stress. As the shear stress increases, the critical radii decrease. However, the two curves cross each other. The twin-boundary, $0.16 \mathrm{~J} / \mathrm{m}^{2}$, energy is taken from Ravelo et al. [34, 37]. Gu et al. [46] reported values using MGPT (modelgeneralized-pseudopotential-theory) of 0.165 and $0.217 \mathrm{~J} / \mathrm{m}^{2}$. For the calculations, we use: $\gamma_{T}=$ $0.2 \mathrm{~J} / \mathrm{m}^{2} ; G=69 \mathrm{GPa} ; \mathrm{b}=0.286 \mathrm{~nm} ;$ and $b_{T}=0.095 \mathrm{~nm}$. For the sake of comparison only, the grain-boundary energy for a random boundary in tantalum is roughly $\gamma_{G B}=0.8 \mathrm{~J} / \mathrm{m}^{2}$, approximately one third of the free surface energy [43]. It is interesting to observe that there is a transition from twinning to shear loop formation for $r_{c T}=3.6 \mathrm{~nm}$. This is consistent with the MD results of Fig. 13, because the critical size observed by MD is a $5 \mathrm{~nm}$ radius. The above is a purely energetic argument and does not take kinetics into account. For instance, delayed kinetics might explain that MD planar defects just before the transition are somewhat larger than model predictions. Still, this transition happens within tens of ps in our simulations, and might be even faster at lower strain rates, where dislocations generally dominate over twinning. 
Figure 15a shows the load-penetration curve obtained by MD. The absence of a horizontal plateau corresponding to the first pop-in, with the emission of the first dislocations (present in Fig. 1) is due to the fact that MD simulations are displacement controlled, whereas nanoindentation experiments are load controlled. Nevertheless, it is possible to identify the sequence of events observed in MD, starting from the first emission of planar defects at a load of $\sim 1.3 \mu \mathrm{N}$. There is a significant difference in the radius of the MD penetrator $(25 \mathrm{~nm})$ and the experimental one (greater than $150 \mathrm{~nm}$ ). The load vs. penetration curves for three crystal orientations are shown in Figure 15b. The radius of the indenter is smaller $(10 \mathrm{~nm})$ than in Fig. 15a and therefore direct comparisons cannot be made. The [001] crystal has the highest 'pop-in' load.

There are significant differences with the measured curves; the 'pop-in' loads in Fig. 1 and Fig. 15b are different by a factor of 100: the plastic plateau occurs at loads between 60 and $200 \mu \mathrm{N}$ in Figure 1 and the onset of plasticity takes place between 0.6 and $2 \mu \mathrm{N}$ in Fig. 15b. The computed hardness from MD is consequently much higher than the experimental one: $13.3 \mathrm{GPa}$ vs. 3.5 GPa. This can be attributed to several causes, including the existence of dislocations in the experimental materials, differences in strain rate, scale effects, and the difference in the radius of indenter $(10 \mathrm{~nm}$ in the $\mathrm{MD}$ calculations and $\sim 100 \mathrm{~nm}$ in the experimental measurements). For instance, Comley et al. [47] experimentally showed a large increase in Ta strength with strain rate [47]. Despite these differences, similar to the ones obtained by Alcalá et al. [28], we find that the ratio of penetration depth to indenter radius at which the first pop-in occurs is $\sim 0.2$ for both simulations and experiments.

\subsection{Calculation of dislocation densities}

Ashby's concept of geometrically necessary dislocations [48] was applied by Nix and Gao [7] to derive an indentation size effect model in which the plastic deformation of the surface was correlated to the emission of dislocations from the surface. It assumes that they are contained in a hemispherical of radius $\mathrm{R}_{1}$, and volume $\mathrm{V}$, below the indented region. This is shown in Fig. 16.

For a conical indenter:

$$
\tan \theta=\frac{h}{a}=\frac{b}{s}
$$


where $\theta$ is the angle of the indented surface, $a$ is the radius of the imprint, and $h$ is the residual plastic depth. The number of geometrically necessary dislocations is $h / b$, where $b$ is the Burgers vector in the two-dimensional geometry, and $s$ is the spacing between individual slip systems on the indented surface. The Burgers vector in the two-dimensional geometry is related to $b_{\langle 111\rangle}$ by the cosine of the angle between [100] and [111]: $1 / \sqrt{3}$. If $\lambda$ is the total length of the dislocation loops injected, then in a differential ring of radii $r$ and $r+d r$, one obtains:

$d \lambda=2 \pi r \frac{d r}{s}=2 \pi r \frac{h}{b a} d r$

Yielding a total loop length of

$\lambda=2 \pi \frac{h}{b a} \int_{0}^{a} r d r=r \frac{h a}{b}$

The hemispherical volume, as defined by Nix and Gao [7], is a function of the contact radius

$V=\frac{2}{3} \pi a^{3}$

The density of geometrically necessary dislocations then is:

$\rho_{G N D}=\frac{\lambda}{V}=\frac{3 h}{2 b a^{2}}=\frac{3}{2 b h} \tan ^{2} \theta$

This model was later extended by Swadener et al. [49]. Later, Durst et al. [50] found that the hemispherical plastic volume is in fact larger than this prediction. They defined a factor, $f$, ranging from 1-3.5, that increases the volume and hence reduces the density of geometrically necessary dislocations:

$V=\frac{2}{3} \pi(f a)^{3}$

$\rho_{G N D}=\frac{\lambda}{V}=\frac{3 h}{2 b a^{2} f^{3}}=\frac{3}{2 b h f^{3}} \tan ^{2} \theta$

For an experimental indentation depth of $100 \mathrm{~nm}$ and an initial $f=1$, Eq. 18 yields a density of geometrically necessary dislocations equal to:

$\rho_{\mathrm{GND}}=1.9 \times 10^{16} \mathrm{~m}^{-2}$

Choosing $f=1.9$ after Durst et al. [50], the achieved density of geometrically necessary dislocations is:

$\rho_{\mathrm{GND}}=2.6 \times 10^{15} \mathrm{~m}^{-2}$ 
This value is very close to the dislocation density measured from TEM: $(1.2 \pm 0.5) \times 10^{15}$ $\mathrm{m}^{-2}$. It must be pointed out that the factor $f$ can vary in the range of 1 to 3.5 , corresponding to $\rho_{\mathrm{GND}}$ in the range of $1.7 \times 10^{16} \mathrm{~m}^{-2}$ to $3.9 \times 10^{14} \mathrm{~m}^{-2}$. To compare with experiments and the analytical models, the dislocation lengths in our simulations were computed by means of the Dislocation eXtraction Algorithm (DXA) [40]. Using the DXA [40], one can then choose a given volume containing those dislocations to obtain dislocation densities. Using the same $f$ factor, $f=1.9$, the radius of the chosen hemispherical volume is $31 \mathrm{~nm}$, for the sample after unloading (residual depth $6 \mathrm{~nm}$ ). This radius of the hemispherical volume guarantees that all the dislocations are contained within the chosen volume, and this is less than half the box depth. The resulting dislocation density is $\rho=7 \times 10^{15} \mathrm{~m}^{-2}$. This value might decrease due to thermally activated dislocation reactions, at times much longer than what can be covered by MD simulations. Thus, considering the uncertainties of measurement and calculations results for experiment, model and simulations are in good agreement.

\section{Summary and Conclusions}

We present a multi-approach study of nanoindentation of tantalum as a model BCC metal, using experiments, molecular dynamics (MD) simulations, and analytical models. Our results can be summarized as follows:

- Experimental loading curves for [001], [011] and [111] indentation directions show distinct pop-ins where the material is deformed plastically. Atomic microscopy scans image the dislocation pile-ups which occur at these pop-ins on the loading curves for all three orientations.

- Large-scale MD simulations show plastic deformation starting with twins, which transform into shear loops, which in turn transform into prismatic loops. The transition from planar faults to dislocations is explained by an analytic energetic model, which agrees well with MD.

- A new mechanism for the transformation of an expanding shear loop into a prismatic loop is identified by MD. The screw components of the shear loop cross slip and pinch out a prismatic loop in a 'lasso' action.

- The geometrically necessary dislocation densities calculated from the three methods are in reasonable agreement. Analytical calculations predict a density in the range $2.6 \times 10^{15}$ - 
$1.9 \times 10^{16} \mathrm{~m}^{-2}$; for MD the predicted value is $\rho=7 \times 10^{15} \mathrm{~m}^{-2}$. Calculations from experimental TEM images show a dislocation density of $1.2 \times 10^{15} \mathrm{~m}^{-2}$. The higher simulation value might be due to lack of thermally activated recovery in MD.

- Simulated pile-ups for [001], [011] and [111] follow crystallographic orientations and form on the diagonal $<110\rangle$ orientations. The average experimental pile-up height for Ta [100] is $h_{p}=4-7 \mathrm{~nm}$ for a penetration depth of 30-35 nm. The height of MD pile-ups is $\mathrm{h}_{\mathrm{p}}=2.5-4.5 \mathrm{~nm}$ for a penetration of $8 \mathrm{~nm}$.

\section{Acknowledgments}

This research was supported by the UC Research Laboratories Grant (09-LR-06-118456MEYM) and a National Laser Users Facility (NLUF) Grant (PE-FG52-09NA-29043). We have a great debt of gratitude to J. Bezares who helped us with the nanoindentation experiments. Tragically he lost his life shortly before completing his doctorate. This research would not have been possible without him. We thank Prof. A. Hodge for her assistance in the experimental part of the program and for essential advice. The transmission electron microscopy was conducted at the ShaRE User Facility, which is sponsored at the Oak Ridge National Laboratory by the Division of Scientific User Facilities, US Department of Energy. We also thank E. Hahn for help preparing three figures. E.M.B. thanks grants from PICT-PRH-0092 and SeCTyP-UN Cuyo. 


\section{References}

[1] Walley SM. Mater Sci Tech 2012;1228:1044.

[2] Armstrong RW, Elban WL, Walley SM. Int J Mod Phys B 2013;1:79.

[3] Armstrong RW, Elban WL. Mater Sci Tech 2012;28:1060.

[4] Jaramillo D, Kuriyama S, Meyers MA. Acta Metall Mater 1986;34:313.

[5] Tabor D. The Hardness of Metals. Oxford: The Clarendon Press;1951.

[6] Oliver WC, Pharr GM. J Mater Res 1992;7:1564.

[7] Nix WD, Gao H. J Mech Phys Sol 1998;46:411.

[8] Gao H, Huang Y. Scripta Mater 2003;48:113.

[9] Lilleodden ET, Nix WD. Acta Mater 2011;59:934.

[10] Gerberich WW, Nelson JC, Lilleodden ET, Anderson P, Wyrobek JT. Acta Mater 1996;44:3585.

[11] Zhua T, Li J, Van Vliet KJ, Ogata S, Yip S, Suresh S. J Mech Phys Sol 2004; 52:691.

[12] Li J, Van Vliet KJ, Zhu T, Yip S, Suresh S. Nature 2002;418(6895):307-10.

[13] Van Vliet KJ, Li J, Zhu T, Yip S, and Suresh S. Phys Rev B 67, 104105 2003.

[14] Meyers MA, Chawla KK. Mechanical Metallurgy. New Jersey: Prentice Hall; 1984.

[15] Zhong Y, Zhu T. Comput Methods Appl Mech Eng 2008;197:3174.

[16] Begau C, Hartmaier A, George EP, Pharr GM. Acta Mater 2011;59:934.

[17] Engles P, Ma A, Hartmaier A. Int J Plasticity 2012;38:159.

[18] Lilleodden ET, Nix WD. Acta Mater 2006;54:1583.

[19] Plimpton S. Fast J Comp Phys 1995;117:1.

[20] Paul W, Oliver D, Miyahara Y, Grütter PH. Phys Rev Lett 2013;110:135506.

[21] Fang TH, Chang WY, Huang JJ. Acta Mater 2009;57:3341.

[22] Tsuru T, Shibutani Y, Kaji Y. Acta Mater 2010;58:3096. 
[23] Begau C, Hartmaier A, George EP, Pharr GM. Acta Mater 2011;59:934.

[24] Traiviratana T, Bringa EM, Benson DB, Meyers MA. Acta Mater 2008;56:3874.

[25] Ziegenhain G, Urbassek HM, Harmaier A. JAP 2010;107:61807.

[26] Hagelaar JHA, Bitzek E, Flipse CFJ, Gumbsch P. Phys Rev B 2006; 73: 045425.

[27] Kumar NN, Tewari R, Durgaprasad PV, Dutta BK, Dey DK. Comput Mater Sci 2013;77:260.

[28] Alcalá J, Dalmau R, Franke O, Biener M, Biener J, Hodge A. Phys Rev Letts 2010;56:109.

[29] Li Y, Siegel DJ, Adams JB, Liu XY. Physical Rev B 2003;67:125101.

[30] Lodes MA, Hartmaier A, Göken M, Durst K. Acta Mater 2011;59: 4264-4273.

[31] Sadrabadi P, Durst K, Göken M. Acta Mater 2009;57:1281.

[32] Kelchner C, Plimpton S, Hamilton J. Phys Rev B 1998;58:11085.

[33] Tramontina D, Erhart P, Germann T, Hawreliak J, Higginbotham A, Park N, Ravelo R, Stukowski A, Suggit M, Tang Y, Wark J, Bringa E. High Energy Density Phys 2014;10:9.

[34] Ravelo R, An Q, Germann TC, Holian BL. AIP Conf Proc 2012;1426:1263.

[35] Tang Y, Bringa EM, Remington BA, Meyers MA. Acta Mater 2011;59:1354.

[36] Moriarty JA, Vitek V,Bulatov VV, Yip S. J of Comput-Aided Mater Design 2002;9:99.

[37] Ravelo R, Germann TC, Guerrero O, An Q, Holian BL. Phys Rev B 2013;88:134101.

[38] Tsuzuki H, Branicio PS, Rino JP. Comput Phys Commun 2007;177:518.

[39] Stukowski A. Modelling Simul Mater Sci Eng 2010;18:015012.

[40] Stukowski A, Albe, K. Modelling. Simul Mater Sci Eng 2010;18:085001.

[41] Biener MM, Biener J, Hodge AM, Hamza AV. Phys Rev B 2007;76:165422.

[42] Yao WZ, Krill CE, Albinski B, Schneider HC, You JH, J. Mat Sci 2014, in press.

[43] Murr LE. Interfacial Phenomena in Metals and Alloys. Addison-Wesley, Reading, MA; 1975. 
[44] Hull D, Bacon DJ. Introduction to dislocations, fourth ed. Oxford: Butterworth-Heinemann; 2001.

[45] Meyers MA, Jarmakani H, Bringa EM, Remington BA. Dislocations in shock compression and release, in: Hirth JP, Kubin L (Eds.). Dislocations in Solids. The Netherlands: NorthHolland; 2009.

[46] Gu Y, Chen LQ, Heo TW, Sandoval L, Belak J. Scripta Mater 2012;68:451.

[47] Comley AJ, Maddox BR, Rudd RE, Prisbrey ST, Hawreliak JA, Orlikowski DA, Peterson SC, Satcher JH, Elsholz AJ, Park HS, Remington BA, Bazin N, Foster JM, Graham P, Park N, Rosen PA, Rothman SR, Higginbotham A, Suggit M, Wark JS. PRL 2013;110:115501.

[48] Ashby MF. Phil Mag 1970;21:399.

[49] Swadener JG, Misra A, Hoagland RG, Nastasi A. J Mech Phys Solids 2002;50:343.

[50] Durst K, Backes B, Göken M. Scripta Mater 2005;52:1093. 


\section{Figure Captions}

Figure 1. Load versus penetration for three orientations of monocrystals [100], [110], and [111]. Hardness equal to $3.5,3.5$, and $5 \mathrm{GPa}$ for the three orientations, respectively. The plastic plateau occurs at loads between 60 and $200 \mu \mathrm{N}$.

Figure 2. Nanoindentation of (100), (110) and (111) Ta single crystals using a Berkovich tip at a load of $\sim 1000 \mu \mathrm{N}$. Atomic force microscopy shows plastic deformation pile-ups around the edges of the nanoindentations, the lighter colors around the perimeter corresponding to pile-ups: (a-b) AFM image and depth profile of (100) Ta showing pile-ups around its perimeter with an average pile-up height $h_{p} \sim 6 \mathrm{~nm}$; (c-d) AFM image and depth profile of (110) Ta showing pile-ups around its perimeter with an average pile-up height $\mathrm{h}_{\mathrm{p}} \sim 5 \mathrm{~nm}$; (e-f) AFM image and depth profile of Ta (111) Ta showing pile-ups around its perimeter with an average pile-up height $\mathrm{h}_{\mathrm{p}} \sim 8 \mathrm{~nm}$.

Figure 3. TEM image of defects generated under nanoindented region for [100] crystal; dislocations imaged in two beam conditions. Greater dislocation density below the apex of the penetration is clearly seen. Some damage due to ion beam sample processing is evident in regions far from indentation sites (shown by black arrow).

Figure 4. TEM image of dislocation loops under a nanoindentation in [111] Ta crystal. The deflection in the surface due to nanoindentation shown by arrow. Loops are clearly seen under, and around, the indentation perimeter.

Figure 5. Molecular dynamics simulation of overall evolution of defects (removing perfect atoms) in nanoindented [100] crystal: (a) at a penetration of $1.8 \mathrm{~nm}$, initial loops are clearly seen; (b) at $2.5 \mathrm{~nm}$ the loops are evolving and interacting; (c) prismatic loop formation at $3.2 \mathrm{~nm}$; (d) generation of successive generations of prismatic loops with continued penetration at $4 \mathrm{~nm}$. The color scheme from red to blue qualitatively designates depth variation, with blue being furthest from surface.

Figure 6. Evolution of shear loops into a prismatic loop by cross-slipping and pinching off of screw components for [100] indentation. The color scheme from red to blue designates the distance of defects from the nanoindentation site.

Figure 7. Evolution of shear loops into prismatic loops by cross-slipping and pinching off of screw components, in a "lasso" action during [001] indentation. (a) shear loop emission; (b) cross slip of screw components to different slip planes and curvature formation at edge component of loop; (c, d, e) continued cross-slip of screw components as edge components advance, in a 'lasso' action, pinching off a loop; (f) release of additional prismatic loop and retraction of shear loop. The color scheme from red to blue qualitatively designates depth variation, with blue being furthest from surface.

Figure 8. Dislocation evolution leading to 'lasso' loop formation with generation of prismatic loop by successive cross slip of screw components along planes sharing the same [111] slip direction, during [111] indentation: (a) shear loop emission; (b) cross slip of 
screw components to different slip planes and curvature formation at edge component of loop; (c, d, e) continued cross-slip of screw components as edge components advance, in a 'lasso' action; (f) attraction of screw components and pinching off of loop; (g) release of prismatic loop and retraction of shear loop. The color scheme from red to blue qualitatively designates depth variation, with blue being furthest from surface.

Figure 9. Triangular, hexagonal, and dodecahedral prismatic loops forming in BCC metals.(Figs. $\mathrm{b}$ and $\mathrm{d}$ from Tang et al. [35]). The color scheme from red to blue designates the distance of defects from the void surface location.

Figure 10. Formation of pile up in indentation along [001]; (a) loops forming along four $\{111\}$ directions; (inclined, top, and side views of pile up; (b) Top view of the residual pile up after indenter removal. The pattern is in agreement with AFM experimental results and corresponds to the activation of four $\{111\}$ systems; (c) Side view of the pile-up. The summits are located in a height range from [2.5 - 4.5] nm. The color scale in (b) and (c) range from blue, sample surface, to red, pile-up summit.

Figure 11. Formation of loops and pile ups for indentation (loading) along (a) [011] and (b) [111]; (a) top view of complete loops forming along [1-1-1] and [1-11] directions; half loops forming on [11-1] and [-1-11] directions which are contained in (011); (b) top view of complete loops forming along [11-1], [-111], and [1-11] (loops forming in [111] not seen). (c,d) pileups for loading in [011] and (b) [111]. The color scale in (c) and (d) ranges from blue, sample surface, to red, pile-up summit at $1.5 \mathrm{~nm}$.

Figure 12. (a) Initial stages of plasticity below indenter; (b) shear stress under indenter, just before nucleation of defects; blue indicates maximum shear, just below the surface at the contact point; (c) Hertzian calculation of normalized maximum shear stress, $\tau_{\max } / P$, as a function of normalized depth, $\varsigma_{a}=z / a$. Maximum for $\mathrm{z} \sim 0.66 \mathrm{a}$.

Figure 13. Formation of planar defects and their evolution into perfect dislocation loops as they expand. (a) Planar faults are shown with a radius of $\sim 5 \mathrm{~nm}$. As they expand, they react, forming perfect dislocation loops in (c) and (d). Penetration depth $h$ marked at top of each figure. The color scheme from red to blue designates the vertical distance of defects from the nanoindentation site.

Figure 14. Analytical model for the nucleation of (a) dislocation loop and (b) twin, under the effect of shear stress $\tau$; (c) critical radius as a function of applied shear stress for dislocation loop and twinning. There is a transition at $\mathrm{r}=3.6 \mathrm{~nm}$, somewhat smaller than found in MD simulations, possibly due to kinetic effects.

Figure 15. (a) MD load-penetration curve for loading and unloading. Target contains about 30 million atoms; radius of indenter: $20 \mathrm{~nm}$; velocity of penetration: $34 \mathrm{~m} / \mathrm{s}$. As the pop-in event takes place, the onset of plasticity occurs with the nucleation of planar defects, as identified by Tang [35] and Alcalá et al. [28]. Plasticity continues with the expansion of shear loops, which later interact and form prismatic loops. After each emission of prismatic loops, shear loops 
develop and evolve, again interacting and producing a new set of prismatic loops. Hardness was calculated to be $13.3 \mathrm{GPa}$, much higher than the experimental value for [100] of $3.5 \mathrm{GPa}$; (b) load for [001], [011] and [111] for a smaller sample (6 million atoms) and indenter radius (10 $\mathrm{nm})$.

Figure 16. Schematic showing geometrically necessary dislocations forming under nanoindentation. (a) Treatment as in Nix and Gao [7] for a conical indenter; (b) terraces due to dislocation slip; (c) treatment as in Swadener et al. [49] for a spherical indenter, using expanded volume. 
Figures

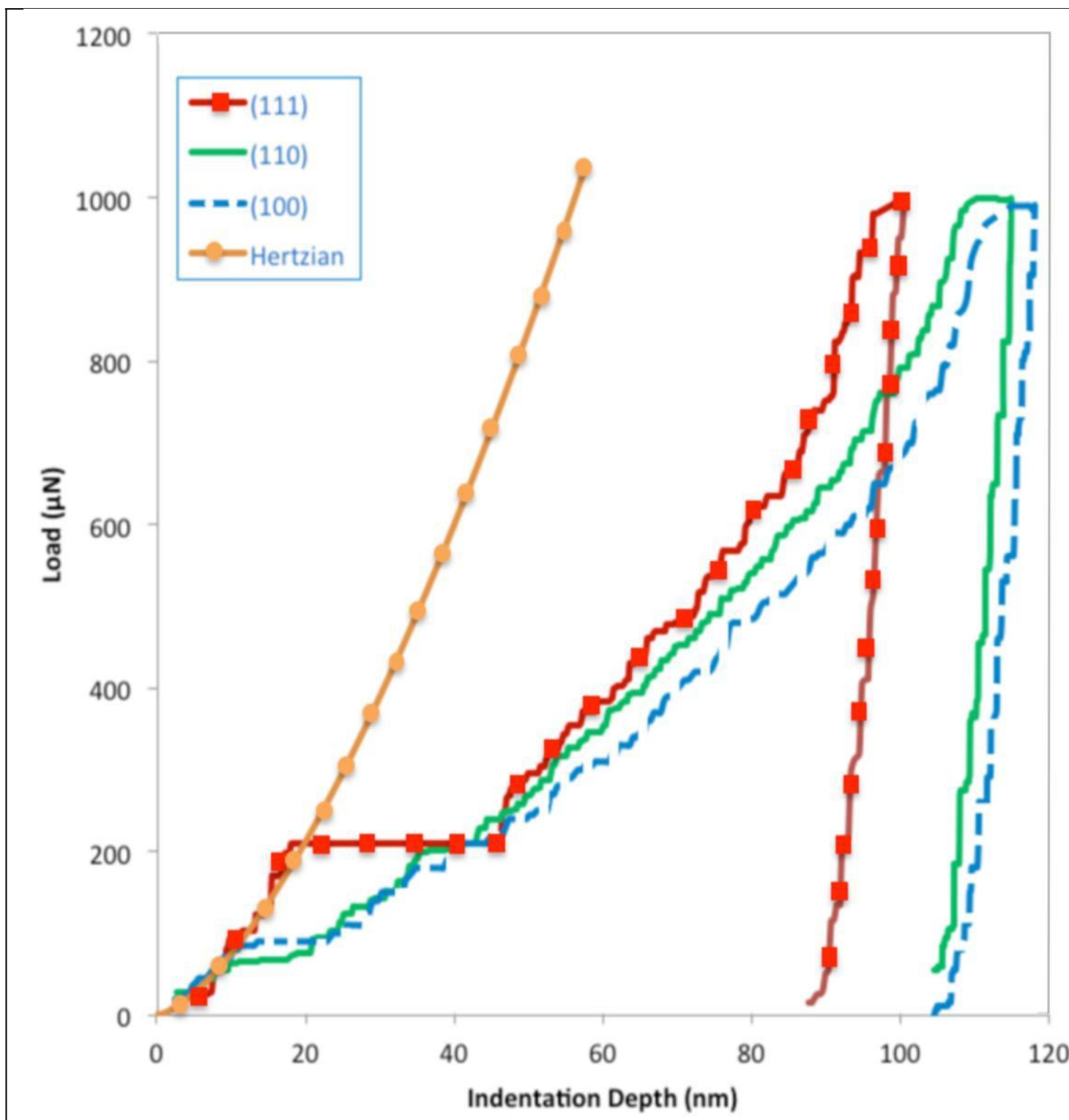

Figure 1. Load versus penetration for three orientations of monocrystals [100], [110], and [111]. Hardness equal to 3.5, 3.5, and $5 \mathrm{GPa}$ for the three orientations, respectively. The plastic plateau occurs at loads between 60 and $200 \mu \mathrm{N}$. 

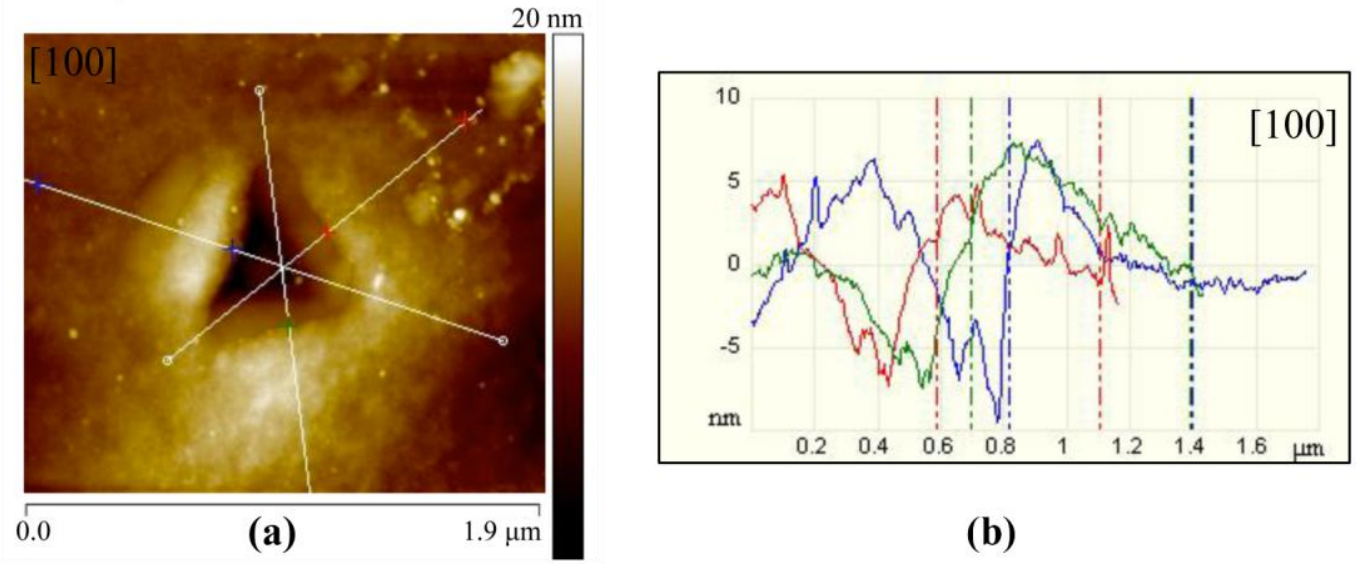

(b)
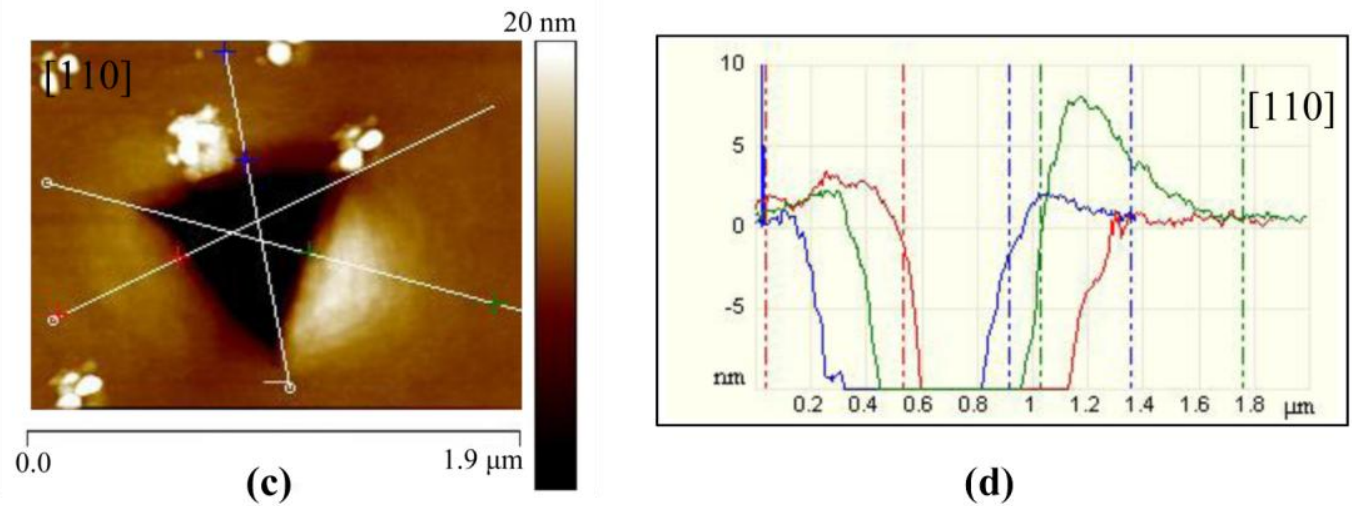

(d)
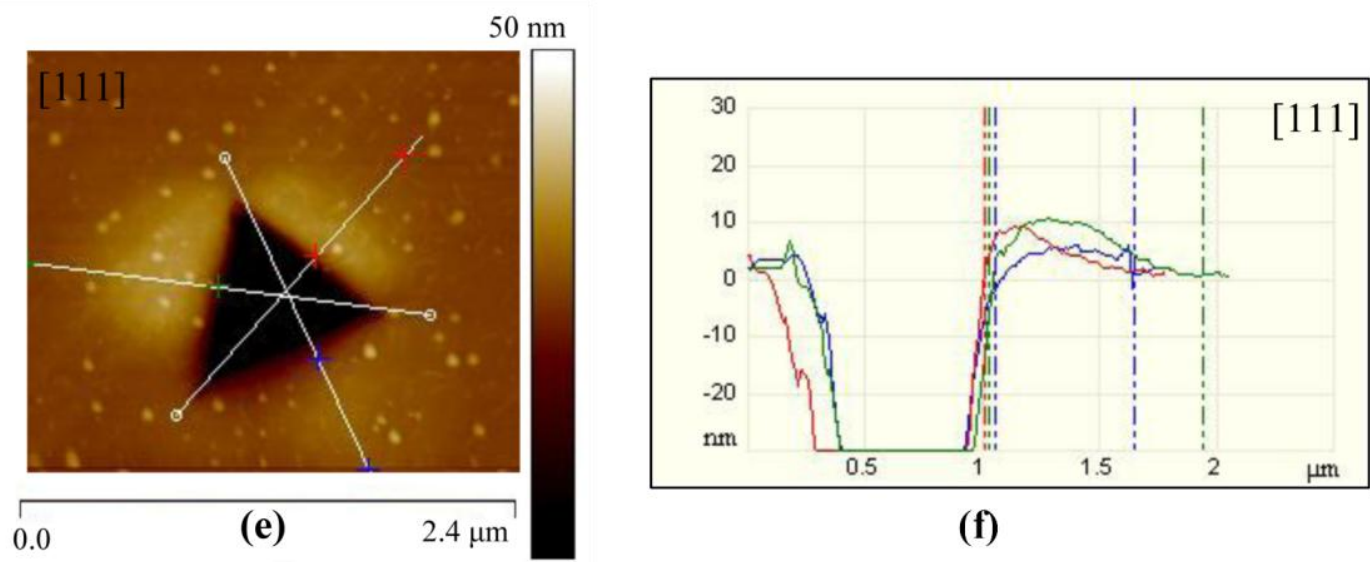

(f)

Figure 2. Nanoindentation of [100], [110] and [111] Ta single crystals using a Berkovich tip at a load of $\sim 1000 \mu \mathrm{N}$. Atomic force microscopy shows plastic deformation pile-ups around the edges of the nanoindentations, the lighter colors around the perimeter corresponding to pileups: (a-b) AFM image and depth profile of [100] Ta showing pile-ups around its perimeter with an average pile-up height $h_{p} \sim 6 \mathrm{~nm}$; (c-d) AFM image and depth profile of [110] Ta showing pile-ups around its perimeter with an average pile-up height $\mathrm{h}_{\mathrm{p}} \sim 5 \mathrm{~nm}$; (e-f) AFM image and depth profile of Ta [111] Ta showing pile-ups around its perimeter with an average pile-up height $\mathrm{h}_{\mathrm{p}} \sim 8 \mathrm{~nm}$. 


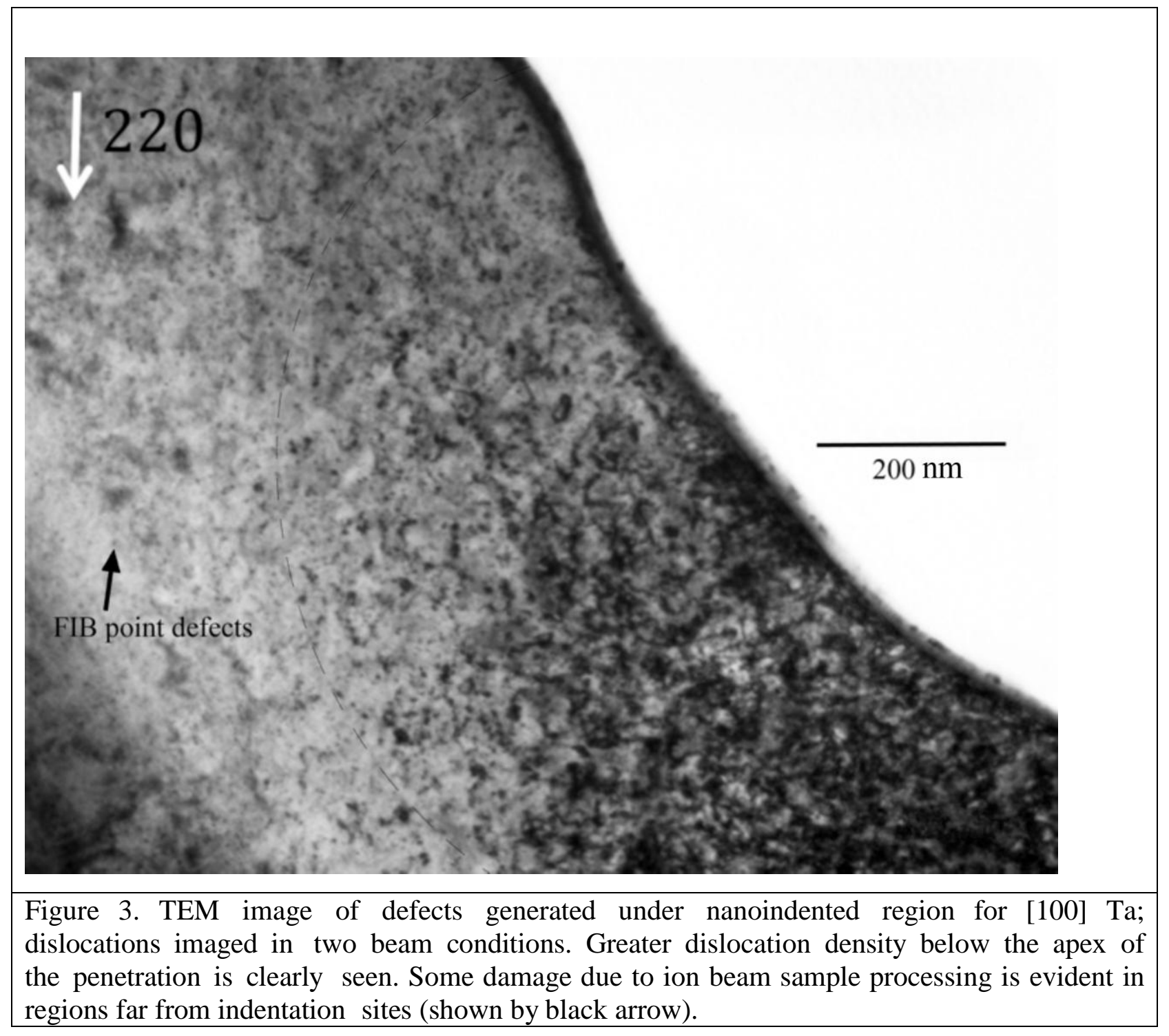




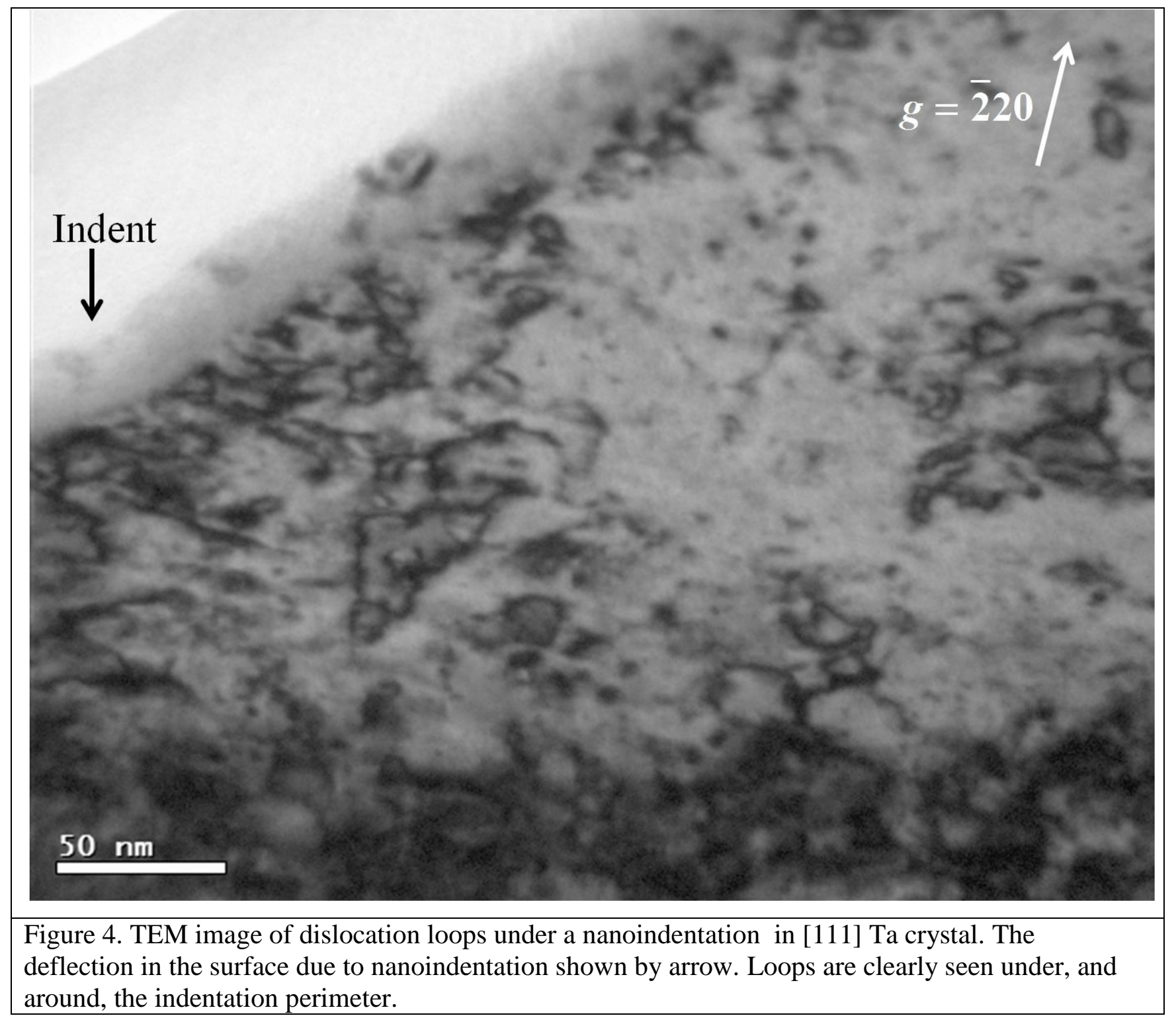




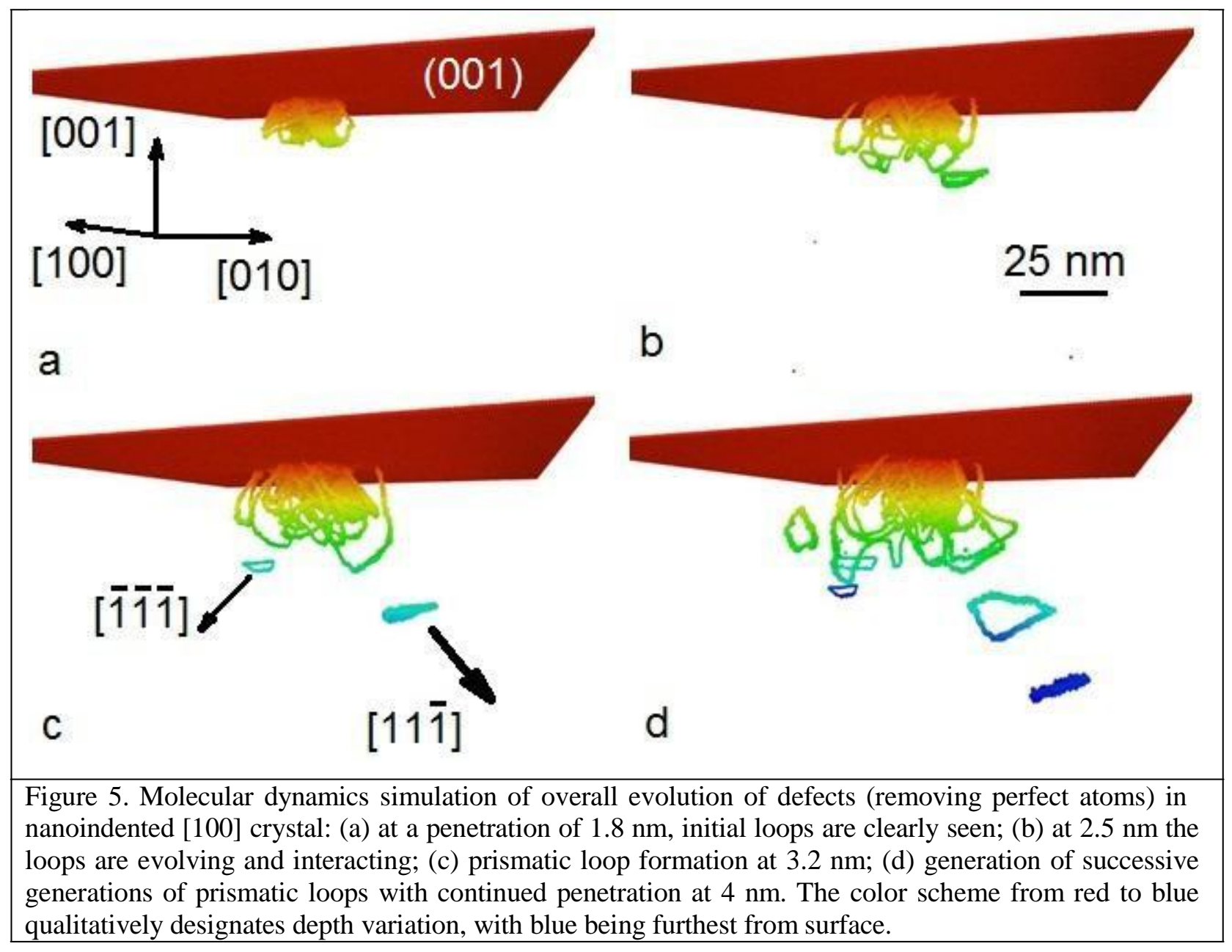




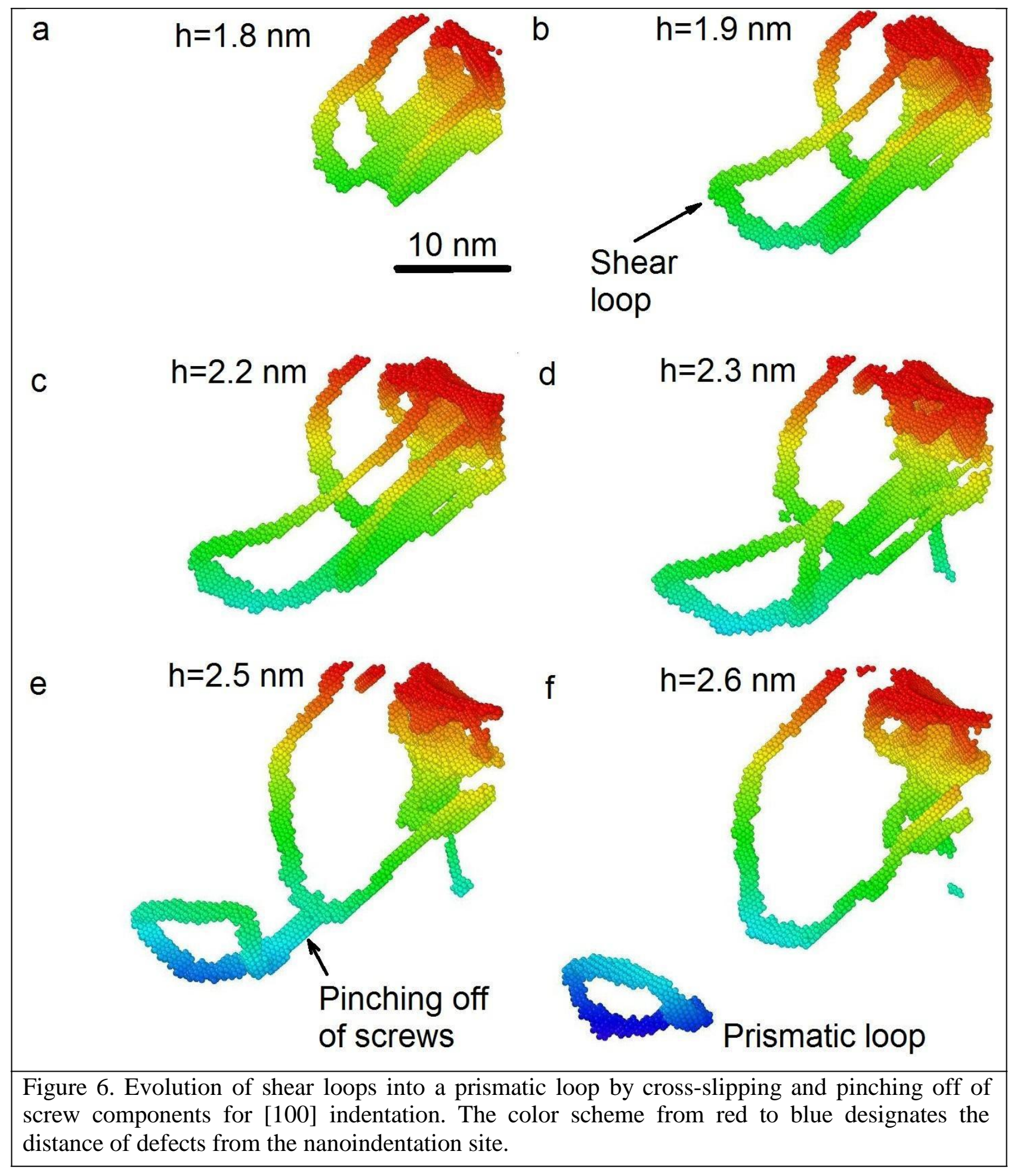




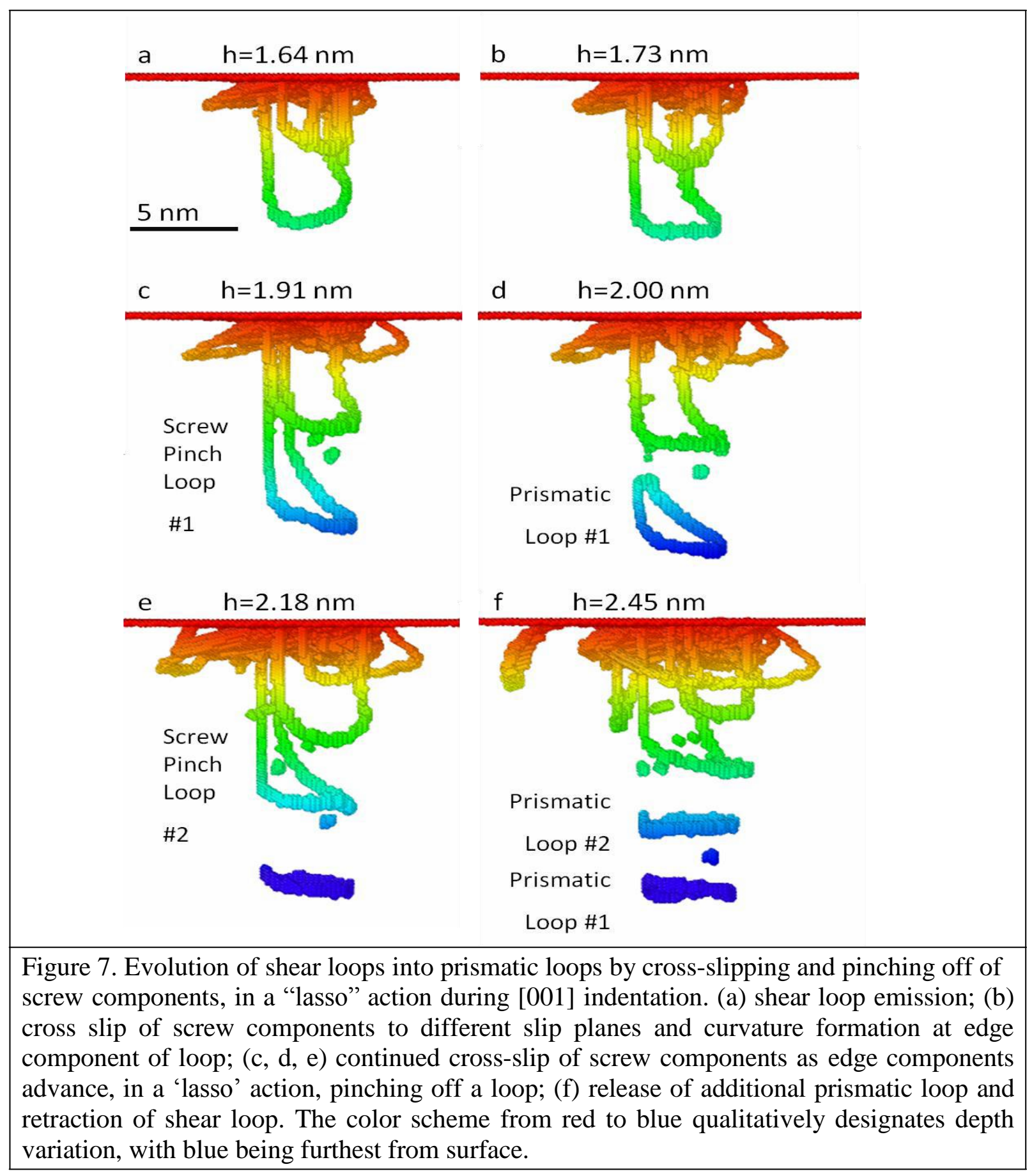




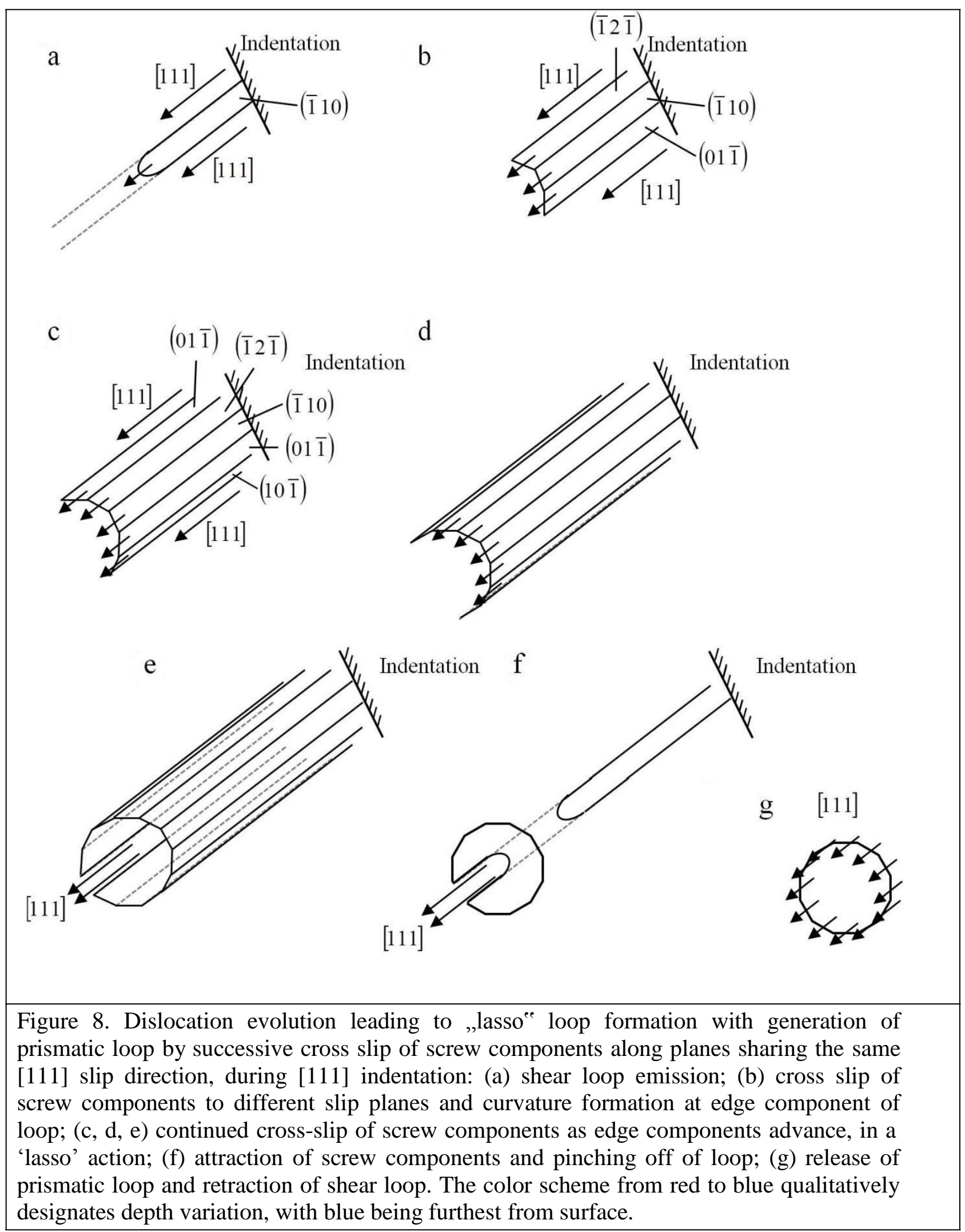




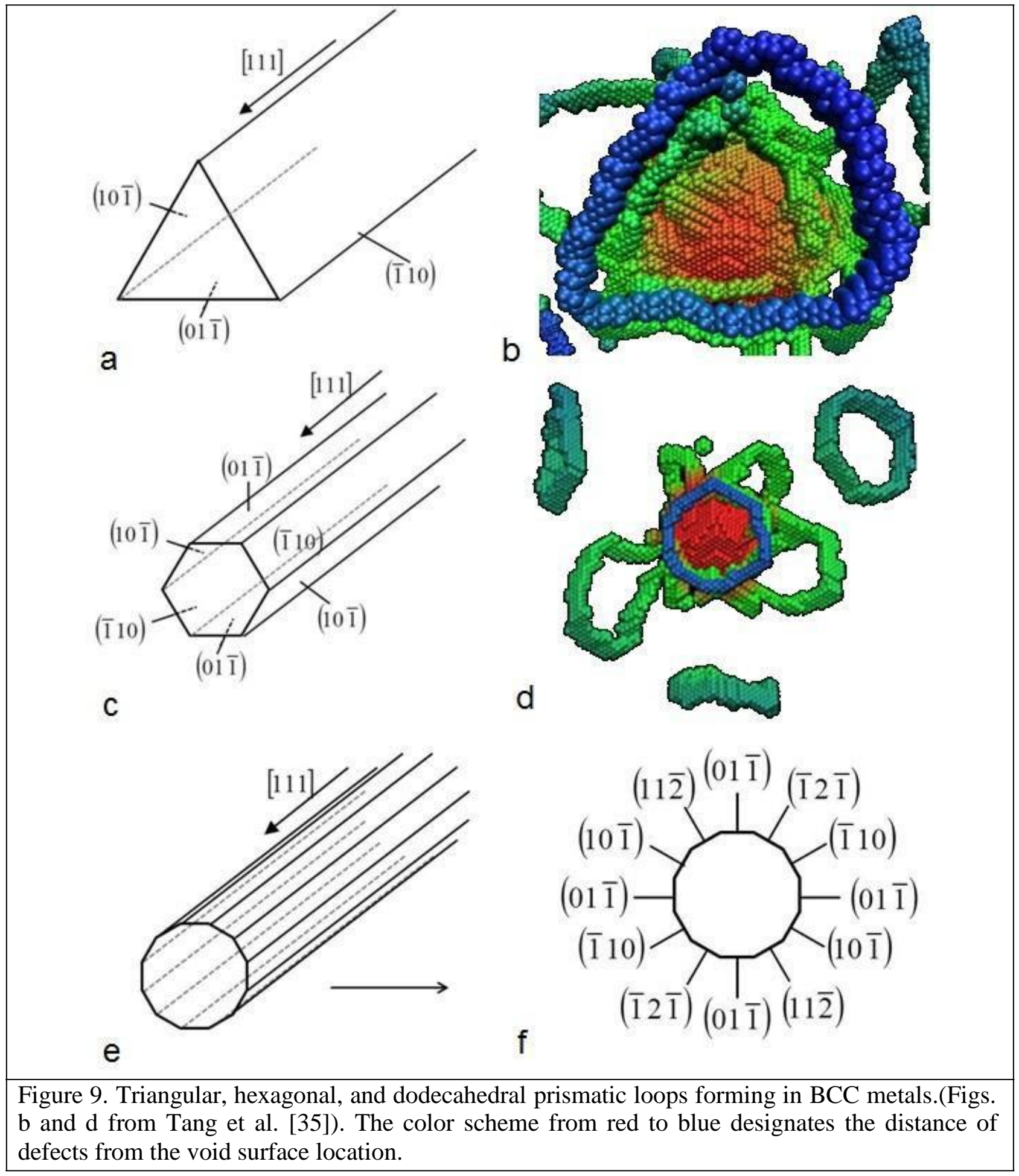



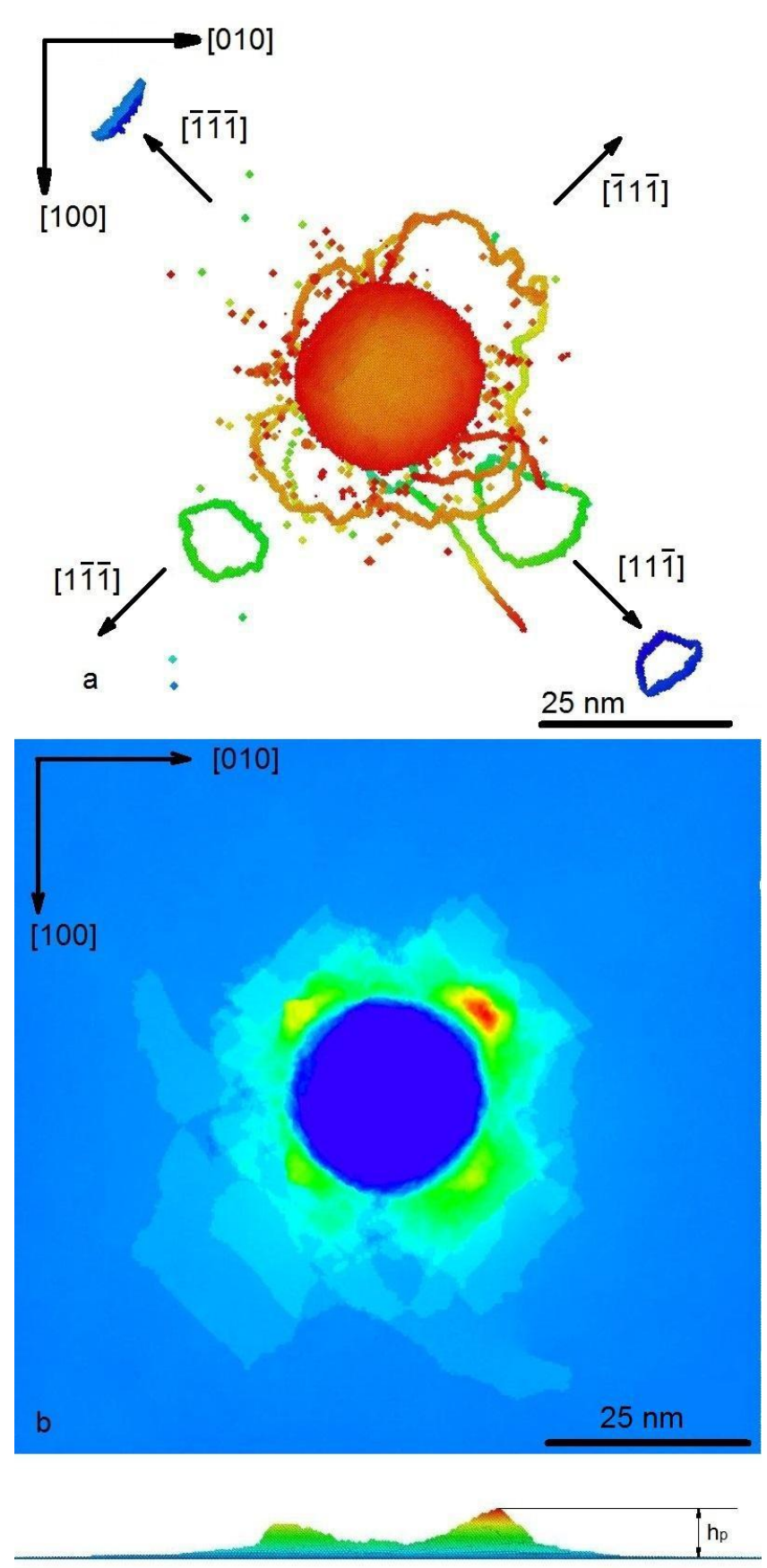

c

$25 \mathrm{~nm}$

Figure 10. Formation of pile up in indentation along [001]; (a) loops forming along four $\{111\}$ directions; (inclined, top, and side views of pile up; (b) Top view of the residual pile up after indenter removal. The pattern is in agreement with AFM experimental results and corresponds to the activation of four $\{111\}$ systems; (c) Side view of the pile-up. The summits are located in a height range from [2.5 - 4.5] nm. The color scale in (b) and (c) range from blue, sample surface, to red, pile-up summit. 


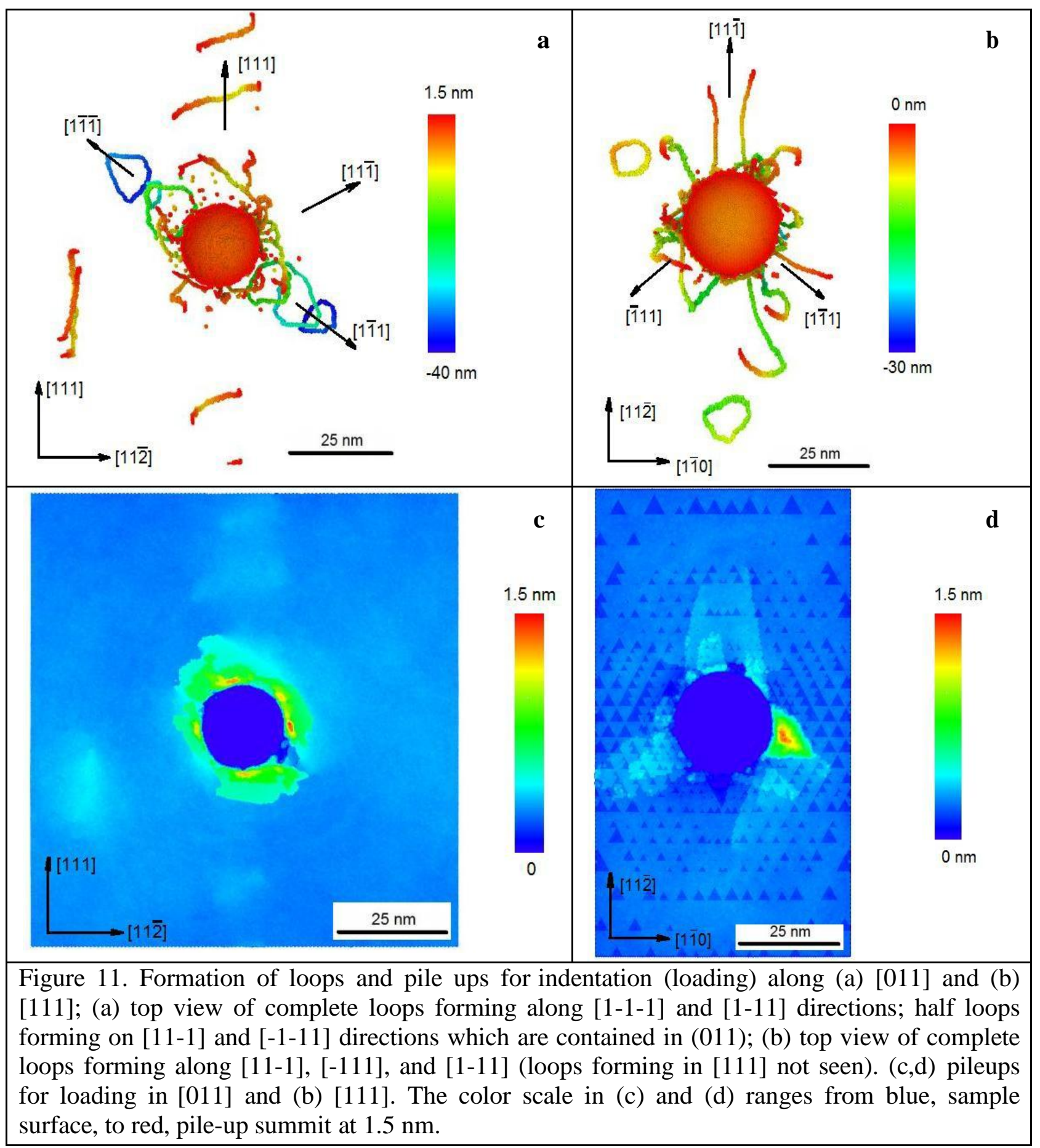




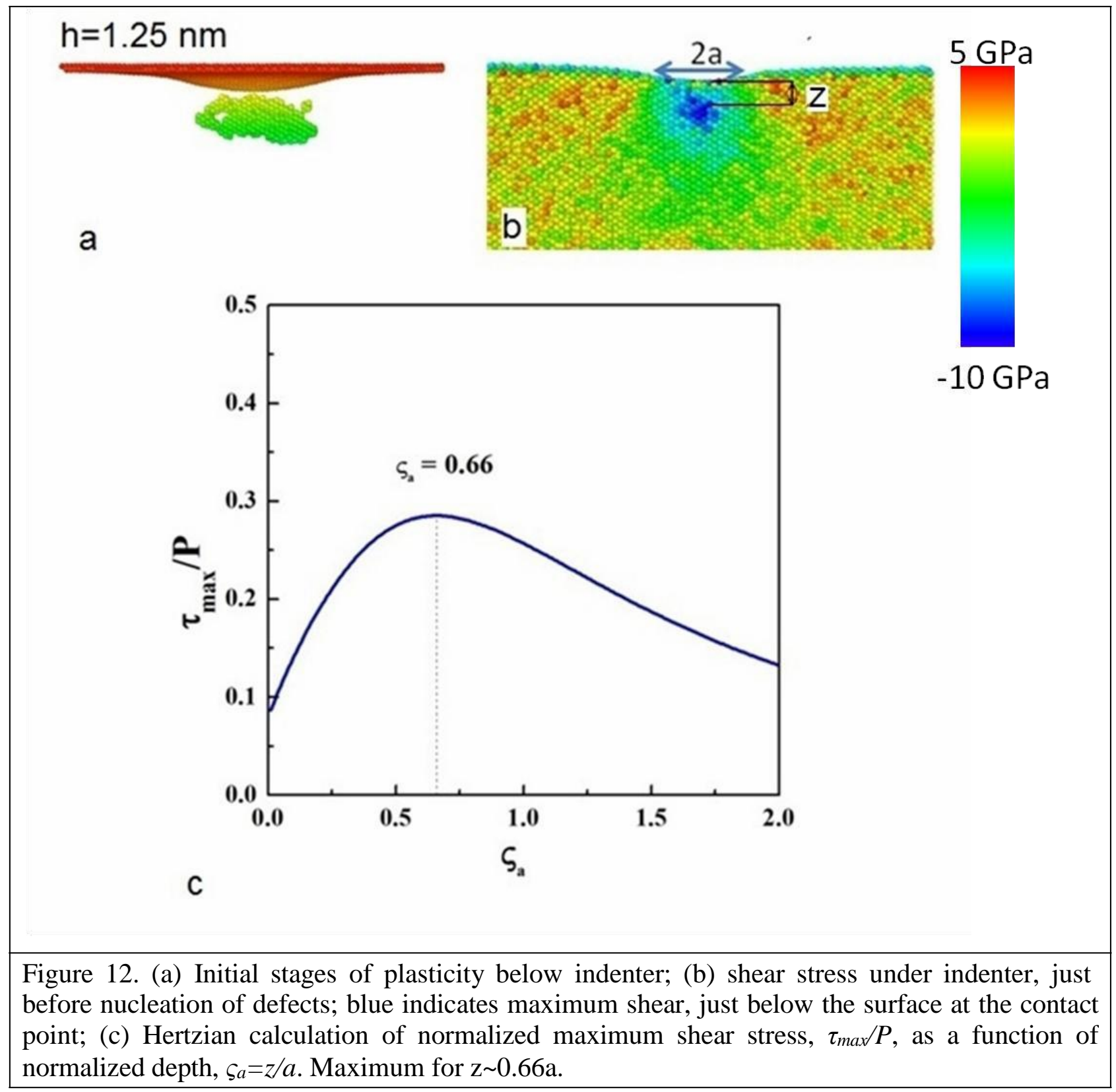




$$
\mathrm{h}=1.3 \mathrm{~nm}
$$

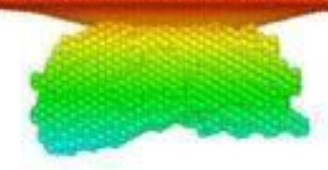

a

\section{$10 \mathrm{~nm}$}

$\mathrm{h}=1.6 \mathrm{~nm}$

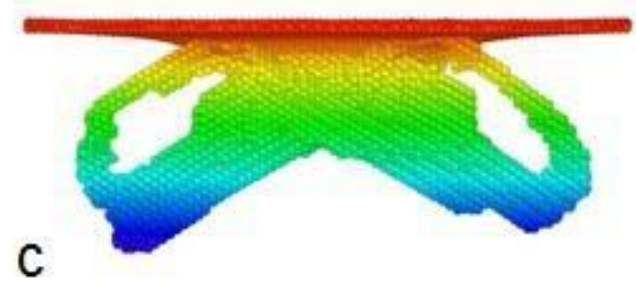

\section{$\mathrm{h}=1.45 \mathrm{~nm}$}

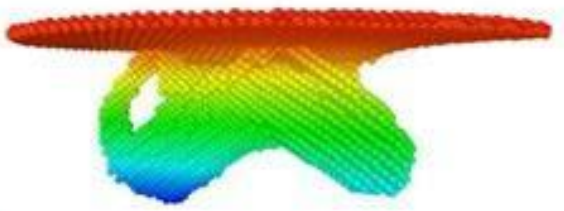

b

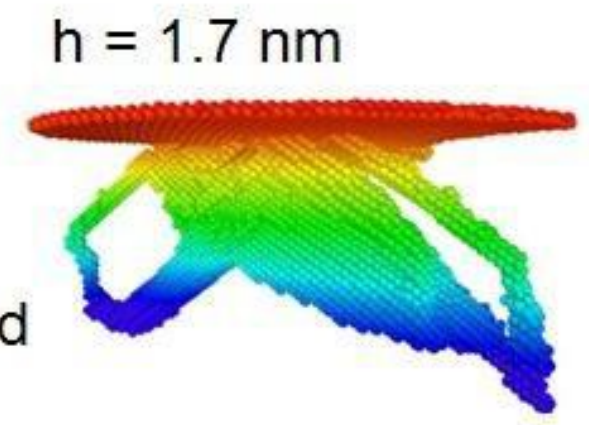

Figure 13. Formation of planar defects and their evolution into perfect dislocation loops as they expand. (a) Planar faults are shown with a radius of $\sim 5 \mathrm{~nm}$. As they expand, they react, forming perfect dislocation loops in (c) and (d). Penetration depth $h$ marked at top of each figure. The color scheme from red to blue designates the vertical distance of defects from the nanoindentation site. 

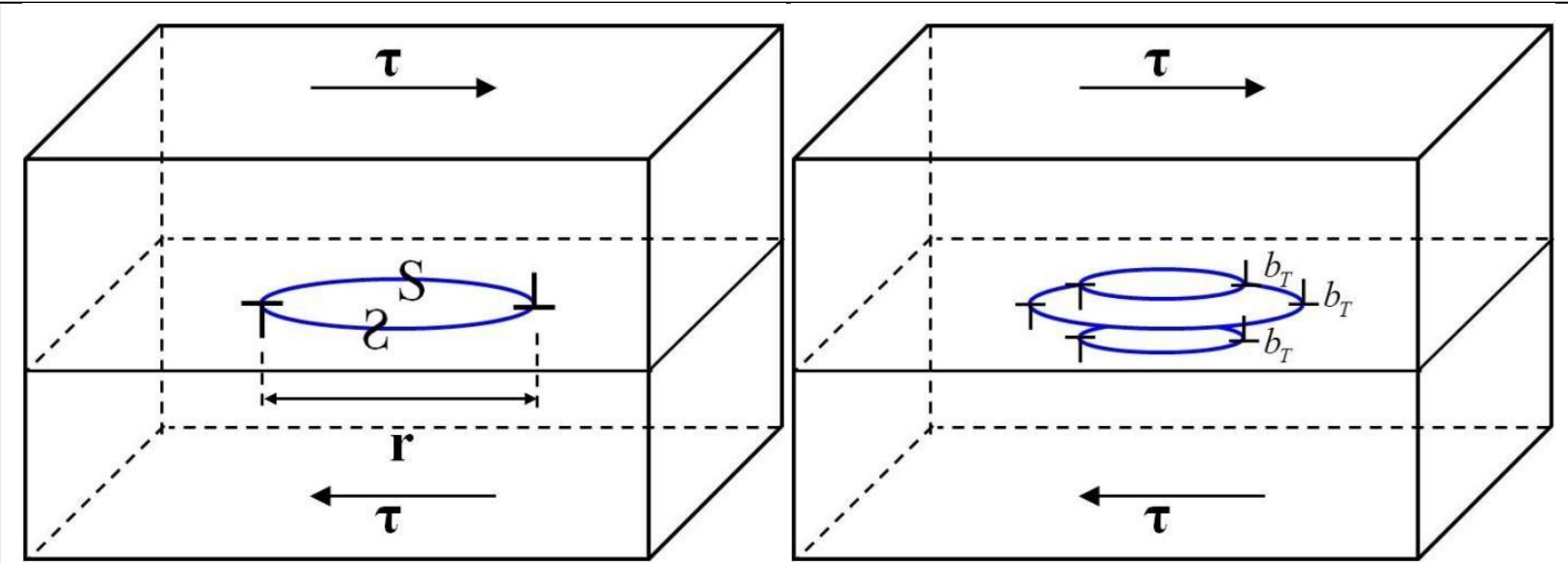

a

b

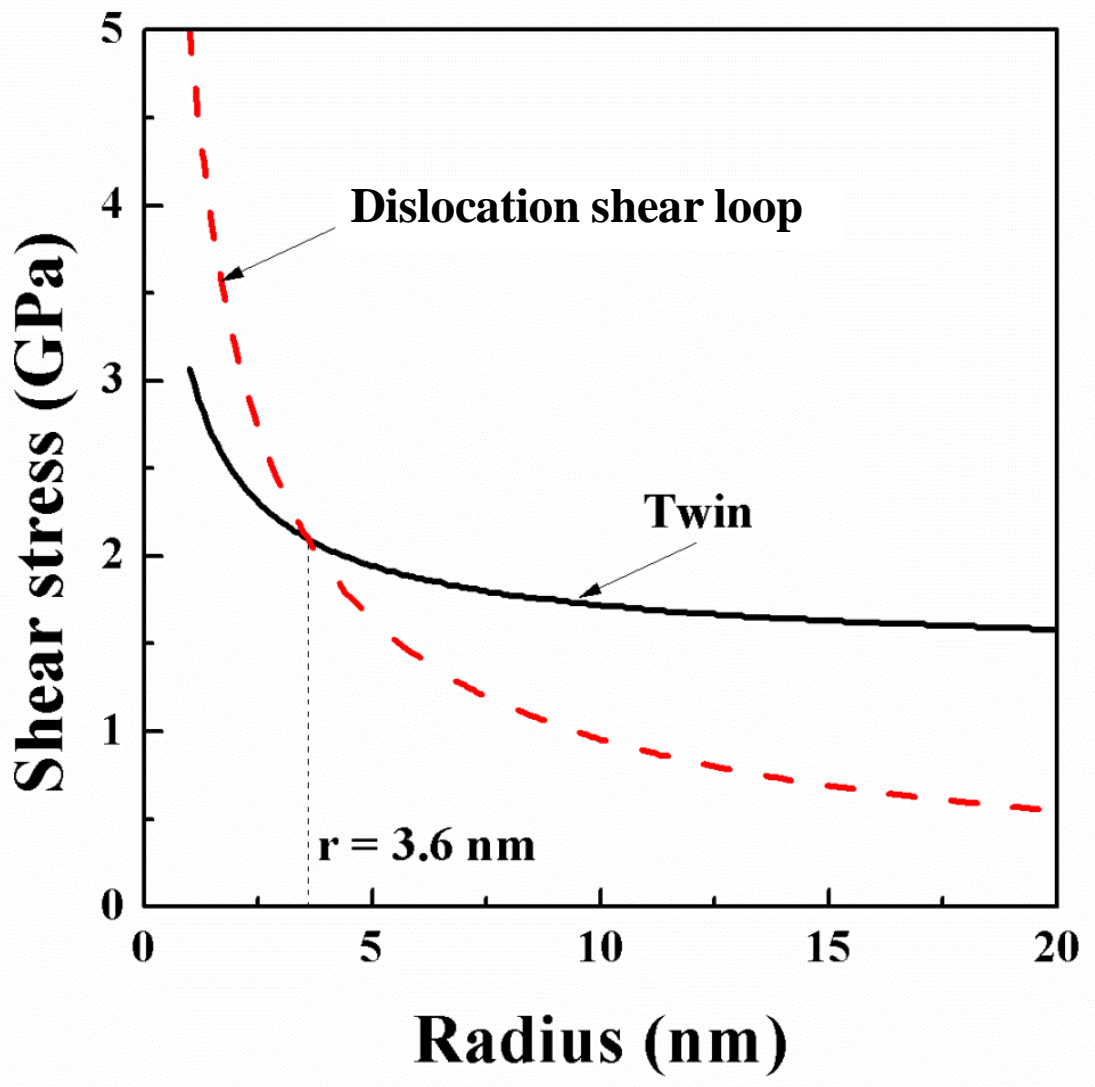

c

Figure 14. Analytical model for the nucleation of (a) dislocation loop and (b) twin, under the effect of shear stress $\tau$; (c) critical radius as a function of applied shear stress for dislocation loop and twinning. There is a transition at $\mathrm{r}=3.6 \mathrm{~nm}$, somewhat smaller than found in MD simulations, possibly due to kinetic effects. 


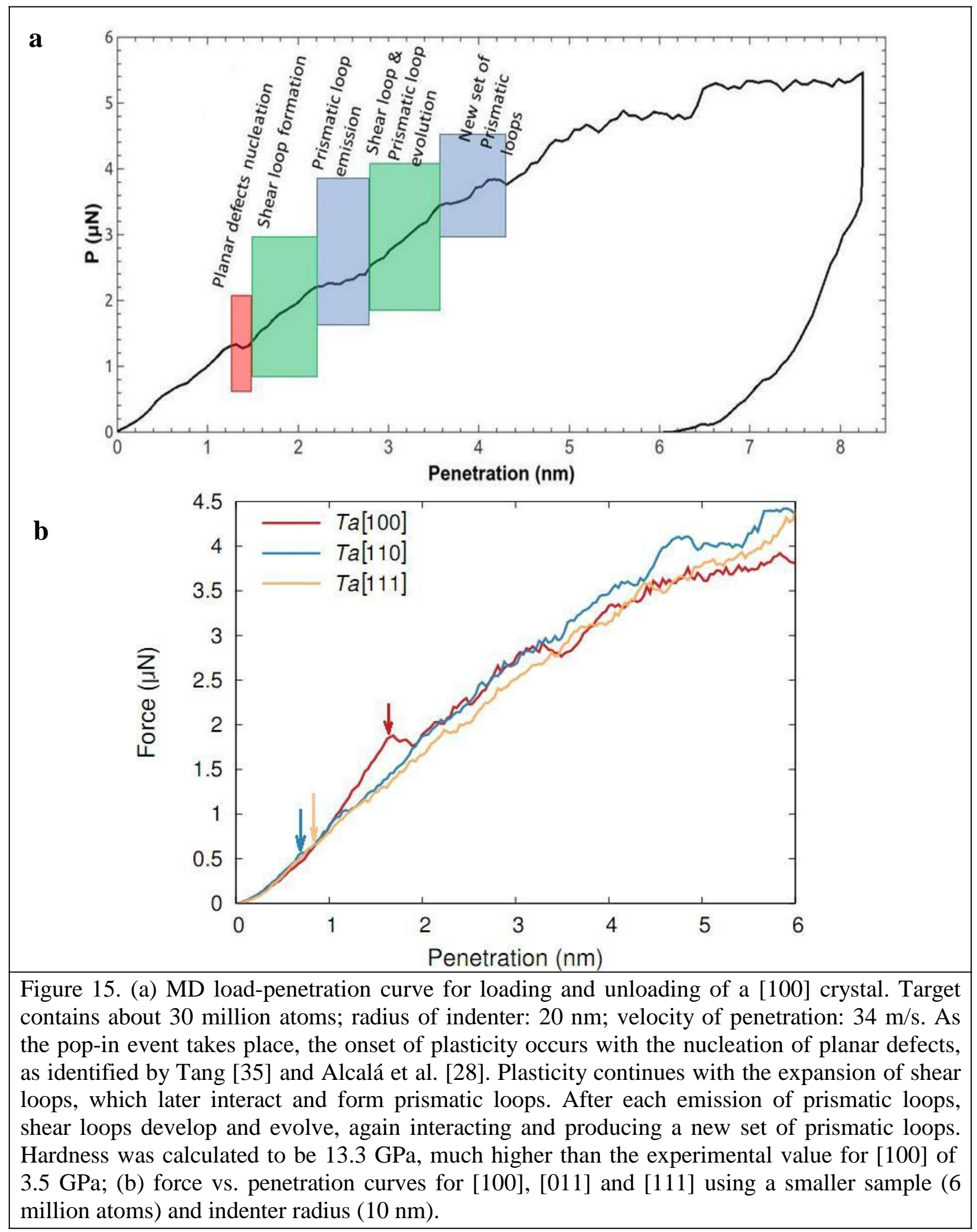




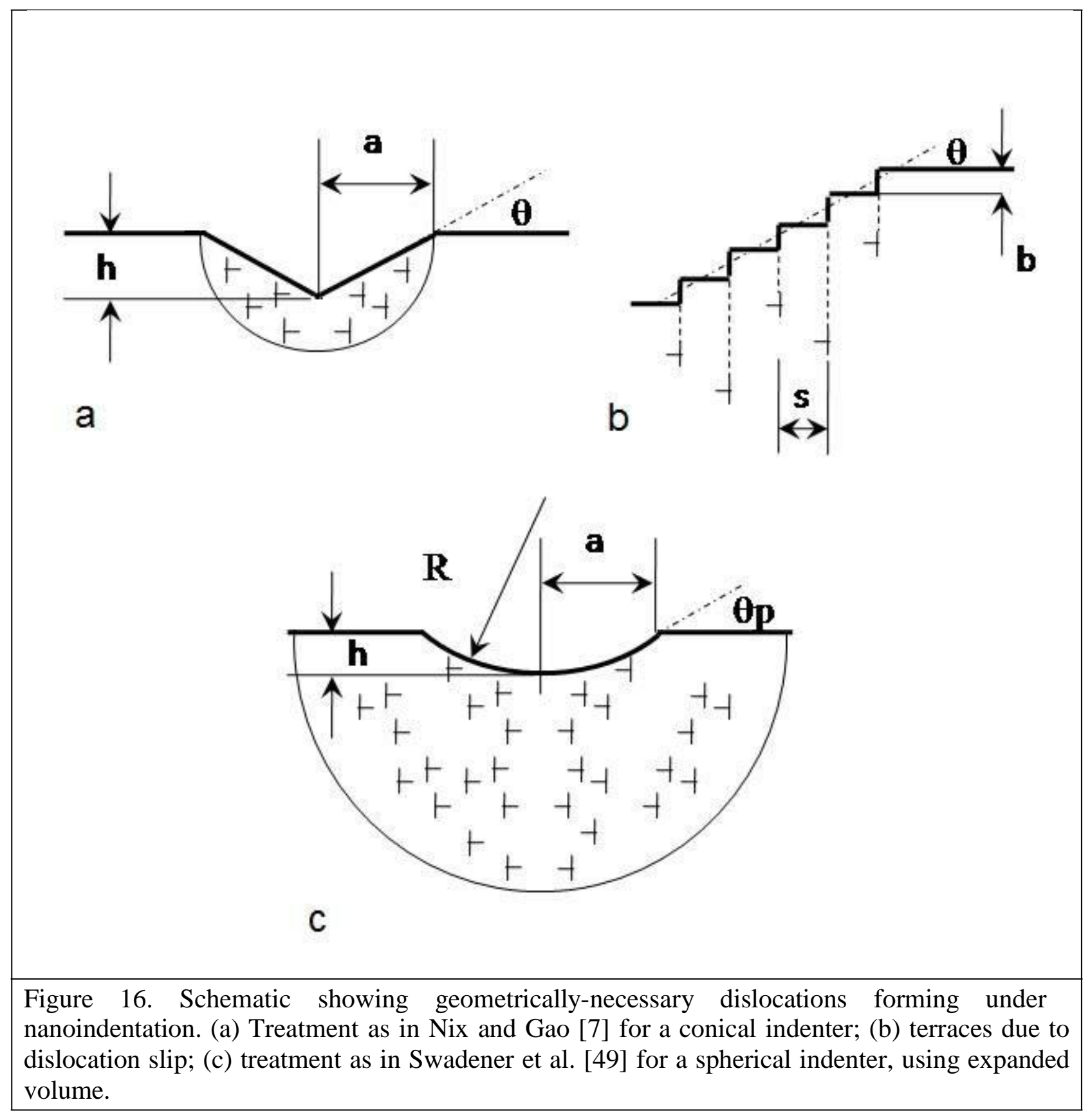

